\title{
Aeromonas hydrophila infection induces Toll-like receptor 2 (t/r2) and associated downstream signaling in Indian catfish, Clarias magur (Hamilton, 1822)
}

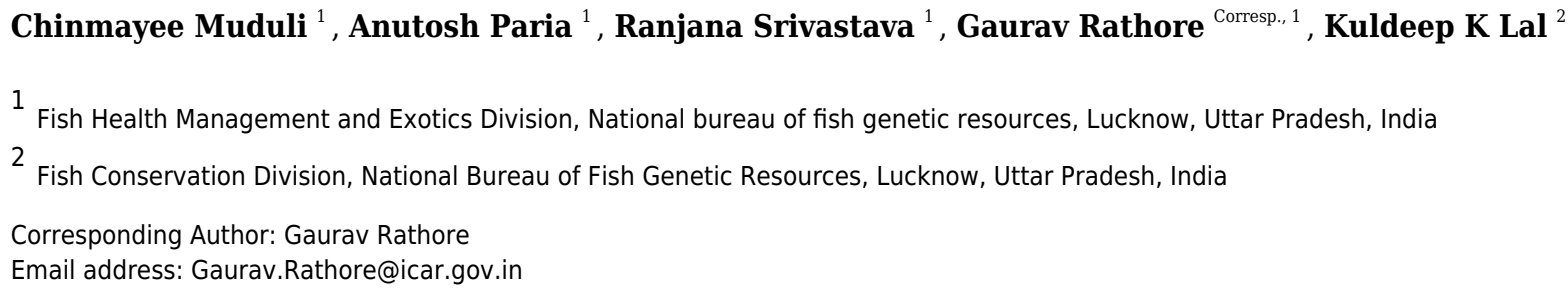

Motile Aeromonas septicaemia (MAS), caused by Aeromonas hydrophila, is one of the most significant bacterial disease responsible for mortality in Indian catfish, Clarias magur, a potential aquaculture species in the Indian subcontinent. In fish, innate immunity elicited by pathogen recognition receptors (PRRs) plays an important role in providing protection against bacterial infection. Information on PRRs including Toll-like receptors (t/rs) and their response to bacterial pathogens remains unexplored in magur. Toll-like receptor 2 (t/r2), a phylogenetically conserved germ-line encoded PRR recognizes specific microbial structure and trigger MyD88-dependent signaling pathway to induce release of various cytokines responsible for innate immune response. In the present study, t/r2 gene of magur was characterized and downstream signaling was studied following challenge with $A$. hydrophila. The full-length cDNA of magur t/r2 ( $m t / r 2)$ comprised of $3066 \mathrm{bp}$ with a single open reading frame of 2373 bp encoding 790 amino acids having a theoretical pl value of 6.11 and molecular weight of $90 \mathrm{kDa}$. Structurally, it comprised of signal peptide (1-42aa), one leucine-rich repeat region (LRR) at N-terminal (LRR1-NT: 50-73 aa) and C-terminal (LRR-CT: 588-608 aa), twenty LRRs in between, one trans-membrane (Tm) domain (609-631aa) followed by cytoplasmic TIR domain (670-783aa). Phylogenetically, $m t / r 2$ is closely related to pangasius and channel catfish. Highest basal expression of $m t / r 2$, myd88 and $i l-1 \beta$ in spleen, $n f-k b$ in anterior kidney was observed. Lowest basal expression of $m t / r 2$ in skin and myd88, nf-kb and $i l-1 \beta$ in muscle was detected. Significant up-regulation of $m t / r 2$ and downstream expression occurred at 3, 8, $24 \mathrm{~h}$ post infection to $A$. hydrophila in important immune organs such as liver, spleen, intestine and kidney. These findings highlight the vital role of $t / r 2$ in eliciting innate immune defence against $A$. hydrophila infection. 
1 Aeromonas hydrophila infection induces Toll-like receptor $2(t / r 2)$ and associated

2 downstream signaling in Indian catfish, Clarias magur (Hamilton, 1822)

3

4 Chinmayee Muduli ${ }^{1}$, Anutosh Paria ${ }^{1}$, Ranjana Srivastava ${ }^{1}$, Gaurav Rathore ${ }^{*}$, Kuldeep K Lal ${ }^{2}$

$5 \quad{ }^{1}$ Fish Health Management and Exotics Division, ICAR-National Bureau of Fish Genetic

6 Resources (NBFGR), Lucknow - 226 002, India

$7 \quad{ }^{2}$ Fish Conservation Division, ICAR-National Bureau of Fish Genetic Resources (NBFGR), Canal

8 Ring Road, Lucknow - 226 002, India

9 *Corresponding Author: Gaurav Rathore

10

11

12

E-mail: Gaurav.Rathore@,icar.gov.in

\begin{abstract}
Motile Aeromonas septicaemia (MAS), caused by Aeromonas hydrophila, is one of the most significant bacterial disease responsible for mortality in Indian catfish, Clarias magur, a potential aquaculture species in the Indian subcontinent. In fish, innate immunity elicited by pathogen recognition receptors (PRRs) plays an important role in providing protection against bacterial infection. Information on PRRs including Toll-like receptors (tlrs) and their response to bacterial pathogens remains unexplored in magur. Toll-like receptor $2(t \operatorname{lr} 2)$, a phylogenetically conserved germ-line encoded PRR recognizes specific microbial structure and trigger MyD88-dependent signaling pathway to induce release of various cytokines responsible for innate immune response. In the present study, tlr2 gene of magur was characterized and downstream signaling was studied following challenge with A. hydrophila. The full-length cDNA of magur tlr2 (mtlr2) comprised of $3066 \mathrm{bp}$ with a single open reading frame of $2373 \mathrm{bp}$ encoding 790 amino acids having a theoretical pI value of 6.11 and molecular weight of $90 \mathrm{kDa}$. Structurally, it comprised of signal peptide (1-42aa), one leucine-rich repeat region (LRR) at N-terminal (LRR1-NT: 50-73 aa) and C-terminal (LRR-CT: 588-608 aa), twenty LRRs in between, one trans-membrane (Tm) domain (609-631aa) followed by cytoplasmic TIR domain (670-783aa). Phylogenetically, mtlr2 is closely related to pangasius and channel catfish. Highest basal expression of $m t l r 2, m y d 88$ and $i l-1 \beta$ in spleen, $n f-k b$ in anterior kidney was observed. Lowest basal expression of $m t l r 2$ in skin and $m y d 88, n f-k b$ and $i l-1 \beta$ in muscle was detected. Significant up-regulation of $m t l r 2$ and downstream expression occurred at 3,8,24 h post infection to A. hydrophila in important
\end{abstract}


32 immune organs such as liver, spleen, intestine and kidney. These findings highlight the vital role

33 of $t l r 2$ in eliciting innate immune defence against $A$. hydrophila infection.

34 Keywords:

Indian catfish, Clarias magur, Pathogen recognition receptors (PRRs), Toll like receptor 2 (tlr2), Pro-inflammatory cytokine, Aeromonas hydrophila, Gene expression

\section{Introduction}

The innate immune system serves as the first line of defence against any invading pathogen. It plays the vital role in defence system in lower vertebrate like teleosts as it has a limited diversity and potency in adaptive immune system compared to the higher vertebrates (Hikima et al., 2011; Zhu et al., 2013). The initiation and regulation of the innate immunity in vertebrates depends largely on different extracellular and intracellular germ line-encoded pattern recognition receptors (PRRs) (Kawai and Akira, 2010; Takeuchi and Akira, 2010). The PRRs, especially signaling PRRs initiates the immune-signaling cascade following recognition of conserved pathogen-associated molecular pattern (PAMP) and damage-associated molecular pattern (DAMP) released during injury or infection (Mogensen, 2009). Among the major classes of signaling PRRs, toll-like receptors (tlrs) are pivotal in sensing extracellular and intracellular microbial PAMPs. Structurally, tlrs possesses an extracellular domain containing leucine-rich repeats (LRRs) that recognise different PAMP and a toll-interleukin 1 (il-1) receptor (TIR) domain for downstream signaling that guide activation of $n f-k b$, which leads to pro-inflammatory cytokines and chemokines production, complement cascade activation, different types of interferons production, dendritic cell maturation, secretory antimicrobial peptides production and stimulation of the adaptive immune system (Mogensen and Paludan, 2005; Akira et al., 2006). These inflammatory signals released from tlrs are protective measure to ensure elimination of detrimental threat caused by infectious agents as well as to accelerate healing process. tlr2 as a member of tlr family is an important component of innate immune system. Among tlrs, tlr2 is best known as the receptor for sensing several PAMPs such as peptidoglycan, lipoteichoic acid, lipoprotein, lipopeptide found in Gram-positive bacteria, lipoarabinomannan, atypical lipopolysaccharides (LPSs), phenol-soluble modulin, porins, glycolipids, poly I:C, LPS, yeast zymosan, glycosylphosphatidyl inositol from protozoan parasites, and also respond to Gram-negative bacteria Porphyromonas gingivalis, Leptospira interrogans, Edwardseilla 
63

64

65

66

67

68

69

ictaluri, Aeromonas hydrophila, Vibrio parahaemolyticus (Ozinsky et al., 2000; Takeuchi et al., 2001; Liu et al., 2001; Baoprasertkul et al., 2007; Fan et al., 2015; Zhang et al., 2017). To recognise these large panel of PAMPs, TLR2 forms homodimer with itself or heterodimer with TLR1 or TLR6 (Takeuchi et al., 2002; Buwittbeckmann et al., 2006). In teleosts, TLR2 cooperate with TLR1 to recognise LPS, poly I:C, lipopeptide (Zhang et al., 2014), as LPS agonist TLR4 is lost from the genome of most fishes and TLR6 is also absent. tlr2 is expressed on various immune cells including neutrophils, monocytes, dendric cells (O'Mahony et al., 2008). TLR2 pathway affects the chemotaxis, phagocytosis, and cytokine release of neutrophils, a critical effector cell against pathogen invasion (Chena et al., 2019). Upon binding to its cognate ligands, TLR2 then recruit the MYD88 and TIRAP ( $m a l)$ that activate the IRAK(1 and 4)/TRAF6/IKK( $\alpha$ or $\beta$ )/MAPKS cascade, which subsequently leads to the ubiquitination of IKB $\alpha$ and the activation of transcription factor NF-kb or AP-1 to induce the expression of host defense genes and subsequent production of pro-, anti-inflammatory cytokines, chemokines, immunomodulatory molecules (Zhang et al., 2014). In recent years, structure and expression pattern of tlr2 has been characterized in few important fish species including zebra fish (Jault et al., 2004), Japanese flounder (Hirono et al., 2004), channel catfish (Baoprasertkul et al., 2007), grouper (Wei et al., 2011), rohu (Samanta et al., 2012), Antratic teleosts (Varriale et al., 2012), shark (Anandhakumar et al., 2012), miiuy croaker (Xu et al., 2013), yellow croaker (Fan et al., 2015), rainbow trout (Brietzke et al., 2016), tongue sole (Li and Sun, 2016), silvery pomfret (Gao et al., 2016), turbot (Zhang et al., 2016; Liu et al., 2016), grass carp (He et al., 2016; Liao et al., 2017), common carp (Fink et al., 2016), golden pompano (Wu et al., 2018) and Dabry's sturgeon (Tang et al., 2020). These studies have provided background information on genomic structure of $t l r 2$ and also reported expression profile of $t l r 2$ upon stimulation with ligand or bacterial infection. Comprehensively, tlr2 play a vital role in innate immune potentiation against wide array of ligand and pathogen in fish species.

Magur is an economically important fish species in South and South-East Asian countries. The freshwater air-breathing Indian catfish, $C$. magur (genetically distinct from $C$. batrachus) (Devassy et al., 2015) is a promising candidate species for freshwater aquaculture in India next to carp (Sahoo et al., 2016). Magur is prone to bacterial and fungal infection in all phase of farming due to scale-less body and many Gram-negative bacterial pathogens have been reported from magur includes, A. hydrophila, A. veronii, Flavobacterium spp, Edwardsiella 
94 95 96

tarda, E. ictaluri, Pseudomonas spp (Monir et al., 2017; Sharma et al., 2017; Sharma et al., 2018). There are no reports on Gram-positive bacterium causing disease in Indian magur. Among the diseases caused by Gram-negative bacterial pathogens, Motile Aeromonas septicaemia (MAS), caused by A. hydrophila, is one of the major causes of concern for finfish culture including magur catfish culture in the subtropical climatic condition like India. Different life stages of magur are affected by $A$. hydrophila infection and the mortality may go up to 70$80 \%$ during rearing stages whereas $50 \%$ mortality has been recorded in grow-out ponds (Sinha et al., 2014; Sharma et al., 2018).

In magur cat fish, information on PRRs including tlrs is miserably inadequate and their role in eliciting innate immune response to bacterial pathogens remains unexplored. $t l r 2$ is one of the important PRRs and plays a significant role in recognition and immunopotentiation against bacterial pathogens. Therefore, the present study was aimed to generate full-length nucleotide sequence of magur tlr2 (mtlr2) cDNA and study its expression along with its downstream signaling molecules such as myd88, $n f-k b$ and $i l-1 \beta$ in different tissues following $A$. hydrophila infection. In the context of the planned experiment, it is worth noting that the cell wall of $S$. aureus contains abundant quantity of PGN, which is a known ligand of tlr2. Hence, Staphylococcus aureus was used as positive control to validate the induction of tlr 2 . The findings of this study would help in understanding the potential role of $t l r 2$ in mediating innate immunity against $A$. hydrophila infection, so that control strategies can be developed.

\section{Materials and methods}

\subsection{Ethical statement}

This research work was carried out in compliance with the animal welfare laws in India. Guidelines of CPCSEA [(Committee for the Purpose of Control and Supervision of Experiments on Animals), Ministry of Fisheries, Animal Husbandry and Dairying, Department of Animal Husbandry and Dairying, Government of India] on care, treatment and experimental protocol of animals in scientific experimentation on fishes were followed. The animal care and experimental challenge protocol was approved by the Institutional Animal Ethics Committee (NBFGR/IAEC/2019/007) of ICAR- National Bureau of Fish Genetic Resources, Lucknow, India.

\subsection{Experimental fish and maintenance}


Apparently healthy farm bred juvenile of $C$. magur $(\mathrm{n}=120$; size-22.31 $\pm 1.34 \mathrm{~cm}$; weight

126

127

128

129

130

131

132

133

134

135

136

137

138

139

140

141

142

143

144

145

146

147

148

149

150

151

152

153

154

155

$50 \pm 4.54 \mathrm{~g}$ ) were handpicked after drying of magur rearing ponds in ICAR-NBFGR, Lucknow, Uttar Pradesh, India. Fishes were transported and stocked in lab condition in 2000L capacity fiber reinforced plastic (FRP) tank with sandy-clay bed provided with hideouts to avoid cannibalism. In each tank, 40 numbers of fish were kept. Fish were fed with commercially available magur feed (Growel Feed Pvt. Ltd, India)@ 2\% of their body weight twice a day.

Fishes were maintained for one month before carrying out the experiment. Half of the water was exchanged weekly.

\subsection{Cloning of magur tlr2 (mtlr2)}

Initially, to amplify partial cDNA sequence of tlr2 gene, PCR primers (Table 1) were designed from conserved region of the nucleotide sequences of channel catfish, Ictalurus punctatus (GenBank Accession HQ677714.1) and yellow catfish, Tachysurus fulvidraco (KU950711.1) tlr2 gene. The initial partial fragment of $m t l r 2$ was amplified from the spleen cDNA of C. magur. The amplified PCR fragment was cloned into pTZ57R/T vector (Thermo Scientific). The recombinant plasmids containing the PCR amplified fragment was sequenced in both the direction using M13 forward and reverse sequencing primers. Likewise, primers were designed from the overlapping sequences of the newly generated sequences and PCR products were sequenced for obtaining the intermittent sequences. To amplify the full-length $m t l r 2 \mathrm{cDNA}$ sequence, 5' and 3'- cDNA ends were amplified using SMARTer RACE cDNA amplification kit (Clontech, USA) and the specific primers designed from the newly obtained sequences. The amplified PCR products of the cDNA ends were cloned and sequenced as mentioned earlier.

\subsection{Bioinformatics analyses of $m$ tlr 2 sequence}

The sequences of different amplicons amplified in PCR were read by Chromas version 2.6.5 and joined together based on consensus sequences to obtain the complete cDNA sequence of $m t l r 2$. NCBI-BLAST (https://blast.ncbi.nlm.nih.gov/Blast.cgi) search was carried out for obtaining the sequence homology of $m t l r 2$ with that of other reported $t l r 2$. The open reading frame (ORF) and the corresponding protein sequence were identified with the help of ORF finder tool of NCBI (https://www.ncbi.nlm.nih.gov/orffinder/). Multiple sequence alignment of deduced amino acid sequence of mtlr2 with vertebrates' counterparts such as human, zebra fish, common carp, rohu, channel catfish and large yellow croaker was carried out using Clustal Omega (https://www.ebi.ac.uk/Tools/msa/clustalo/) to identify the conserved features and 
156 domains. Signal peptide, transmembrane domain and TIR domain were predicted using the

157 SMART software (http://smart.embl-heidelberg.de/) whilst the remaining LRR motifs were

158 identified manually. LRR domain was also searched in the deduced amino acid sequence for the

159 presence of LxxLxLxxNxL as decribed by (Matsushima et al., 2007). The theoretical isoelectic

160 point $\left(\mathrm{P}_{\mathrm{i}}\right)$ and molecular weight $\left(\mathrm{M}_{\mathrm{w}}\right)$ were computed with protParamExpasy programme

161 (http://web.expasy.org/protparam/). The amino acids were deduced based on cDNA sequences

162 using the translate program found on the ExPASy website. Microsatellites in the $m t l r 2$ sequence

163 were identified using microsatellite repeats finder

164 (http://insilico.ehu.es/mini tools/microsatellites/). Nucleotide and protein blast was carried out to

165 determine identity percentage of $m t l r 2$ with other related fish species. To construct phylogenetic

166 tree, multiple-sequence alignment of $t l r 2$ amino acid sequences of fishes, human, animals was

167 carried out with the help of Clustal Omega. Phylogenetic tree of $t l r 2$ was constructed by

168 neighbour joining method of MEGA 7.0 programme bootstrapped 1000 times using default

169 parameters.

170 2.5. Bacterial strains

171 Our previously isolated Aeromonas hydrophila 9C strain (Muduli et al., 2020) was used 172 as test strain, while Staphylococcus aureus (ATCC 25923) was used as positive control for this

173 experiment. The bacteria were cultured in tryptic-soy broth (TSB) at $37{ }^{\circ} \mathrm{C}$, overnight and total

174 plate count was estimated individually for both the strains before in-vivo challenge.

\section{2.6. In vivo bacterial challenge and sample collection}

176 One hundred twenty numbers $(\mathrm{n}=120)$ of apparently healthy magur $(\sim 50 \mathrm{~g})$ were

177 randomly divided into three groups i.e. negative control (Phosphate buffer saline or PBS

178 injected), treatment (A. hydrophila injected) and positive control (S. aureus). Each group

179 consisted of 40 fish in 1000L FRP tank. Completely randomized design (CRD) was followed to

180 set up the experiment. Before initiation of the experiment, three fish were sampled randomly for

181 bacterial and parasitological examination to ensure that fish were free of bacterial and parasitic

182 infection. During the experimental period, the temperature, dissolved oxygen and $\mathrm{pH}$ were $35 \pm$

$1832^{\circ} \mathrm{C}, 6.8 \pm 0.78 \mathrm{mg} \mathrm{L}^{-1}, 8.2 \pm 0.45$, respectively as measured by a multiparameter water quality

184 meter (Hanna Instruments, Romania), whereas, nitrite and ammonia were estimated to be 0.013

$185 \pm 0.007 \mathrm{mg} \mathrm{L}^{-1}$ and $0.106 \pm 0.02 \mathrm{mg} \mathrm{L}^{-1}$, respectively following APHA (1998) protocol. 
186

187

188

189

190

191

192

193

194

195

196

197

198

199

200

201

202

203

204

205

206

207

208

209

210

211

212

213

214

215

216

For bacterial infection, fishes in treatment group were intra-peritoneally (i.p.) injected with Lethal dose $\left(\mathrm{LD}_{50}\right)$ of $A$. hydrophila at $1 \times 10^{5} \mathrm{cfu} / \mathrm{fish}$ in $100 \mu 1$ of sterile phosphate buffer saline (PBS). Fishes in positive control were intra-peritoneally injected with $S$. aureus at $1 \times 10^{5}$ $\mathrm{cfu} /$ fish in $100 \mu \mathrm{l}$ PBS. The negative control group were injected with $100 \mu 1$ of sterile PBS and kept separately. Thereafter, at 3, 8, 24, 72, 144 hours post-infection (hpi), three fish from bacteria-injected and control group were randomly picked, euthanized in 300mg/L concentration of MS-222 and sacrificed. Tissues such as gill, liver, spleen, intestine and kidney tissue were dissected out in to RNAlater and stored at $-80^{\circ} \mathrm{C}$ until RNA extraction. At the end of the experiment, the survived fishes in A. hydrophila and S. aureus group were kept separately for further studies.

\subsection{Total RNA extraction and cDNA synthesis}

Total RNA was extracted from the tissues using TRI Reagent (Sigma-Aldrich) as per the manufacturer's instructions. Tissue sample of 30-50 mg was homogenized in $1 \mathrm{ml}$ of TRI reagent using lysing matrix D beads $(1.4 \mathrm{~mm})$ in FastPrep homogenization system (MP Biomedicals). RNA pellet was dissolved in diethyl pyrocarbonate (DEPC) treated nuclease-free water and stored at $-80^{\circ} \mathrm{C}$ until further use. RNA concentration and purity were analysed in Denovix UV-Vis spectrophotometer (Denovix). To remove the residual genomic DNA, $1 \mu \mathrm{g}$ total RNA was treated with $1 \mathrm{U}$ of DNase I, RNase free (Thermo Scientific) following manufacturer's protocol. DNase-treated total RNA $(1 \mu \mathrm{g})$ was reverse-transcribed using RevertAid First Strand cDNA Synthesis kit with oligo-dT primer (Thermo Scientific) as per the manufacturer's instructions.

\subsection{Quantitative real-time PCR (qRT-PCR) primers}

The real-time PCR primers were designed for $m t l r 2, m y d 88, n f-k b, I L-1 \beta$ and $e f-1 \alpha$. The details of primers used in real-time PCR were listed in table 1. Before performing qPCR, amplification efficiency was determined. A series of four-fold dilution of cDNA ranging from $2000 \mathrm{ng}$ to $7.8 \mathrm{ng}$ was prepared using cDNA synthesized from pooled spleen tissue of three healthy C. magur. Standard curve, correlation coefficient $\left(R^{2}\right)$ and PCR amplification efficiencies $(E)$ were automatically generated in the ABI StepOne Plus qPCR system based on slope of standard curve produced using four-fold diluted cDNA as template. The formula used to calculate efficiency was as follows $E(\%)=\left(10^{-1 / \text { slope }_{-}}\right) \times 100$ (Kubista et al., 2006). The primers with PCR efficiencies between 90-105\% were used for relative quantification. 
217

218

219

220

221

222

223

224

225

226

227

228

229

230

231

232

233

234

235

236

237

238

239

240

241

242

243

244

245

246

247

\subsection{Basal expression analysis of $m t l r 2, m y d 88, n f-k b, i l-1 \beta$}

Basal expression of $m t l r 2$ and its downstream signaling molecules $m y d 88, n f-k b, i l-1 \beta$

were determined. Twelve different tissues; gill, liver, spleen, intestine, anterior kidney, posterior kidney, stomach, skin, muscle, heart, brain and accessory respiratory organ (ARO) were collected separately from three naive Indian magur $(\sim 50 \mathrm{~g})$. Total RNA was isolated from each sample and cDNA was prepared following the manufactures protocol. Quantitative real-time PCR as mentioned later was carried out. Tissue with lowest expression was used as calibrator with respect to which fold change expression of above genes were calculated using the $2^{-\Delta \Delta \mathrm{Ct}}$ method (Livak et al., 2001). In our earlier experiment on validation of reference genes in $C$. magur, ef-1 $\alpha$ was found to be the most stable reference gene for normalization of relative gene expression by real-time reverse transcription PCR in the context of pathological conditions ( $A$. hydrophila injected and PBS injected) (data unpublished). Therefore, ef- $1 \alpha$ was used as an internal control.

\subsection{0. qRT-PCR}

The qRT-PCR was performed in Applied Biosystem StepOne Plus Real-time PCR system using TB Green ${ }^{\mathrm{TM}}$ Premix Ex Taq ${ }^{\mathrm{TM}}$ II (TAKARA) following manufacture's instruction. Amplification was carried out in $12.5 \mu 1$ reaction volume, comprising $6.25 \mu 1 \mathrm{SYBR}$ green master mix, $0.25 \mu 1$ of ROX, $0.5 \mu 1$ of forward and reverse primer ( $5 \mu \mathrm{M}$ each), $1 \mu 1$ of cDNA (100ng/ul) and $4 \mu \mathrm{l}$ of nuclease-free water. Each sample was run in triplicate, which served as technical replicate under following cycling condition: initial denaturation at $95{ }^{\circ} \mathrm{C}$ for $30 \mathrm{~s}$ followed by 40 cycles of $94{ }^{\circ} \mathrm{C}$ for $10 \mathrm{~s}, 60^{\circ} \mathrm{C}$ for $10 \mathrm{~s}, 72{ }^{\circ} \mathrm{C}$ for $10 \mathrm{~s}$. This was subsequently followed by melt-curve analysis for $15 \mathrm{~s}$ at $95{ }^{\circ} \mathrm{C}$ and $1 \mathrm{~min}$ at $60^{\circ} \mathrm{C}$. A no template control in triplicate was run for each plate. The data in form of threshold cycle $(\mathrm{Ct})$ for each sample was normalized with corresponding ef-1 $\alpha$. The fold-change in expression in the treatment group was compared with that of PBS-injected control group using the $2^{-\Delta \Delta \mathrm{Ct}}$ method (Livak et al., 2001). The data obtained from qRT-PCR analysis from nine technical replicate out of 3 biological replicate were subjected to statistical analysis.

\subsection{Statistical analysis}

All data were expressed as mean \pm standard error of mean (S.E.M). Statistical analysis was performed using SPSS 16.0 software (SPSS Inc., Chicago, USA). The significant difference in fold change expression at different time point within individual tissue was determined with 
248 time point as independent variable and fold change in expression as dependent variable at 95\%

249 significance level using statistical model ANOVA with Tukey post-hoc test $(p<0.05)$. The $p$

250 values significant at $<0.05,<0.01,<0.001$ levels, respectively, compared to controls indicated by

251 different superscripts.

252

253 3. Results

\section{3.1. Characterization of full-length cDNA of $\boldsymbol{m t l r} 2$}

255 Full-length cDNA encoding mtlr2 was amplified from magur spleen cDNA. Initially, two set of 256 primer (2F-3R, 3F-5R primer) yielded 626 bp (Fragment 1) and 1129 bp (Fragment 2) 257 amplification. Further, a new set of internal/nested primer (F3-R3) generated 694 bp (Fr3)

258 overlapping Fragment 1 and 2. Joining these fragments together a long stretch of $1830 \mathrm{bp}$ 259 product was obtained. Cloning, sequencing, and NCBI-BLAST search of $1830 \mathrm{bp}$ cDNA had 260 sequence similarity with other fish $t l r 2$ which confirmed it to be $t l r 2$. The 3'- and 5'- RACE were 261 performed to obtain 3'- and 5'- terminal regions of cDNA. The full-length mtlr2 cDNA was 3066 $262 \mathrm{bp}$, including 5' and 3' un-translated region (UTR) of 113 and $582 \mathrm{bp}$, respectively. The predicted 263 single ORF of mtlr2 was 2373 bp, located between 114-2487 bp nucleotide and encodes 790 264 amino acids with a theoretical pI value of 6.11 and molecular weight $90.095 \mathrm{kDa}$. Structurally, it 265 comprised of a signal peptide (1-42 aa), one leucine-rich repeat region (LRR) at N-terminal (LRR1-NT; 50-73 aa) and C-terminal LRR (LRR-CT; 588-608 aa), twenty LRRs in between Nand C-terminal, one trans-membrane (Tm) domain (609-631 aa) followed by one TIR domain (670-783 aa) (Fig. 1, 2 and 3). The highly conserved motifs were identified in TIR domains, and wide range of diversity was observed within the LRR regions (Fig.3). Two microsatellites were detected in the UTR region of cDNA sequence of $m$ tlr 2 i.e., pentamer penta repeat (GTTTT) ${ }_{5}$ at $69 \mathrm{bp}$ of 3'UTR region and dimer octarepeat $(\mathrm{AC})_{8}$ repeat at $2862 \mathrm{bp}$ of 5'UTR region. One hexamer tri-repeat $(\mathrm{CTGACT})_{3}$ also present in coding region at $215 \mathrm{bp}$ location. Two mRNA instability motifs (ATTTA) were found in the 3'-UTR, which suggest that mtlr2 may be transiently expressed (Fig. 1). The mtlr2 nucleotide sequence was submitted to GenBank database with GenBank ID: MT625141.

\subsection{Structural identity and phylogenetic relationship of $m t l r 2$ with other species}

Similarity comparison of the mtlr 2 nucleic acid sequence with pangasius, channel catfish, and yellow catfish revealed identity percentage of $83.19,79.20$ and 80.69 , respectively at nucleic 
279 acid level. At the deduced amino acid level, the corresponding identity \% was 77.63, 76.4, and

28072.83 , respectively with pangasius, channel catfish, and yellow catfish. With carp and higher

281 vertebrate family, the identity $\%$ of $m t l r 2$ amino acids was $\sim 60$ and $\sim 37$, respectively. The

282 identity of conserved TIR domain of $m t l r 2$ ranged from $89.9 \%$ with pangasius catfish to lowest

$28357.4 \%$ with Japanese flounder. TIR domain of $m t l r 2$ shares highest identity with catfish group

284 i.e. $89.9 \%$ with pangasius. TIR domain of $m t l r 2$ shares identity of $77.5 \%$ with zebrafish, $64 \%$

285 with mouse and $65.2 \%$ with human (Table 2).

286 Phylogenetic tree was constructed to understand the evolutionary relationship of $m t l r 2$

287 with $t l r 2$ reported from other teleosts. Among the fishes, tlr2 representing catfish group

288 including magur, channel catfish, yellow catfish and pangasius were closely related and formed

289 one cluster, and were well separated from carps viz., rohu, mrigal, common carp, grass carp and

290 zebra fish. Within the members of catfish family, mtlr2 branched with pangasius and channel

291 catfish, indicating close evolutionary relationship. Other group of fishes such as trout, bream,

292 flounder, croaker, grouper, and cod formed a separate cluster from carps and catfishes. As

293 oblivious from phylogenetic tree that fish tlr2 are well divergent from other higher animals

294 (cluster-IV) (Fig. 4).

295

296

297

298

299

300

301

302

303

304

305

306

307

308

\subsection{Basal expression of $m t l r 2$ and its downstream molecules}

The qRT-PCR amplification efficiencies $(E)$ of primer sets fell within the suitable experimental range as per MIQE guideline (Bustin et al., 2009) i.e. from $95.81 \%$ to $104.66 \%$; while correlation coefficient $\left(R^{2}\right)$ ranged from 0.989 to 0.998 respectively (Table 3 ). Tissue specific expression of $m t l r 2, m y d 88, n f-k b$ and $i l-1 \beta$ gene in twelve different tissues including gill, liver, spleen, anterior kidney, posterior kidney, intestine, accessory respiratory organ (ARO), stomach, muscle, brain, heart and skin was evaluated by qRT-PCR assay. Their expression in various organs/tissues was normalized with the reference gene $e f-1 \alpha$ and represented as relative

fold changes from the lowest expressing tissue (calibrator). Highest basal expression of $m t l r 2$, $m y d 88$ and $i l-1 \beta$ in spleen, $n f-k b$ in anterior kidney was observed. Lowest basal expression of $m t l r 2$ in skin and myd88, $n f-k b, i l-1 \beta$ in muscle was detected. Although, the tissue-specific expression of mtlr 2 was varied but the expression was at detectable level in all the examined tissue (Fig. 5 A, B, C, D).

\subsection{Inductive expression of $\boldsymbol{m t l r} 2$ in response to $S$. aureus}


Modulations of mtlr 2 gene transcripts were analyzed in gill, liver, spleen, intestine and

310

311

312

313

314

315

316

317

318

319

320

321

322

323

324

325

326

327

328

329

330

331

332

333

334

335

336

337

338

339

kidney by qRT-PCR following i.p. injection of $S$. aureus. As shown in Fig 6, mtlr2 expression was significantly induced in various tissues at different time points.

\subsection{Differential expression of $m t l r 2$ and its downstream signaling molecules in response to}

\section{A. hydrophila infection}

Modulation of $m t l r 2$ expression was studied following $A$. hydrophila infection in gill, liver, spleen, intestine and kidney. The kinetics of up- or down-regulation of the mtlr 2 gene exhibited a similarity of general trend, rising to a high level at 3 to 24 hpi and then falling to the basal level at 72 to 144 hpi, but the up- and down-regulation differed at different time points in different tissues. A significant up-regulation of $m t l r 2, m y d 88, n f-k b$ gene expression was observed in gill, liver, spleen, intestine and kidney within 24 hpi (Fig. 7 A, B, C). Similarly, significantly up-regulated expression of $i l-1 \beta$, a pro-inflammatory cytokine was noticed in gill, liver, spleen, intestine and kidney within 24 hpi (Fig. 7 D).

\section{Discussion}

In the present study, we have identified an orthologue of mammalian tlr2 in Indian catfish, $C$. magur which is a potential aquaculture species in South-East Asia. Two mRNA instability motifs (ATTTA) were found in the 3'-UTR, which suggest that mtlr2 may be transiently expressed. Two mRNA instability motifs (ATTTA) were also reported in turbot (Zhang et al., 2016). The full-length cDNA of $m$ tlr 2 is of $3066 \mathrm{bp}$ and comprised of an ORF of $2373 \mathrm{bp}$ nucleotide in length, encoding 790 amino acid residues. The putative $m t l r 2$ protein possesses the four typical component of $t l r$ families from $\mathrm{N}$-to $\mathrm{C}$-terminus i.e. signal peptide, one LRR each at N- and C- terminal, twenty extracellular LRR domains in between N- and Cterminal, trans-membrane domain and highly conserved cytoplasmic TIR domain. Number of LRR domain in other fishes reported are 9 LRRs in channel catfish (Baoprasertkul et al., 2007), 21 in rohu (Samanta et al., 2012), 20 LRRs in human, zebra fish, channel catfish, common carp (Fink et al., 2016), 19 in turbot (Zhang et al., 2016), 11 in silvery pomfret (Gao et al., 2016), 8 in yellow catfish (Pelteobagrus fulvidraco) (Zhang et al., 2017), 8 in Dabry's sturgeon (Tang et al., 2020). The number of LRR signifies the functional capability to respond various agonists.

Structurally, $m t l r 2$ is closely related to channel catfish $t l r 2$ gene, which also consists of an ORF of $2373 \mathrm{bp}$ encoding protein of 790 amino acids (Baoprasertkul et al., 2007). Our finding on 
340

341

342

343

344

345

346

347

348

349

350

351

352

353

354

355

356

357

358

359

360

361

362

363

364

365

366

367

368

369

370

number of LRRs are similar to finding by Samanta et al. (2012, Fink et al. (2016), Zhang et al. (2016). Also, the genomic structure of a single exon and no intron reported in human, zebra fish, and channel catfish tlr2 (Baoprasertkul et al., 2007), but fugu and Japanese flounder tlr2 gene encoded by 11 and 12 exons, respectively (Oshiumi et al., 2003; Hirono et al., 2004). Whether $m t l r 2$ gene possesses introns or not needs further investigation. Two microsatellites were detected in the UTR of mtlr2 i.e., pentamer pentarepeat (GTTTT) ${ }_{5}$ at 69 bp of 3'UTR and dimer octarepeat $(\mathrm{AC})_{8}$ at $2862 \mathrm{bp}$ of $5^{\prime} \mathrm{UTR}$. One hexamer tri-repeat (CTGACT) ${ }_{3}$ also present in coding region. In channel catfish, two microsatellites were identified in $t l r 2$ sequence i.e. (AT) 9 repeat at $632 \mathrm{bp}$ and an $(\mathrm{AC})_{21}$ repeat at 3606bp (Baoprasertkul et al., 2007). The identified microsatellite will allow the mapping of $t$ tr 2 gene and future quantitative trait loci (QTL) analysis in C. magur.

The phylogenetic tree constructed using different vertebrate $t l r 2$ with that of $m t l r 2$ showed a possible four major clades; carps, catfishes, other teleosts, and higher vertebrates. Phylogenetically, mtlr2 is closely related to pangasius and channel catfish. The close phylogenetic relationship indicates the functional similarities. Study on channel catfish $\mathrm{tlr} 2$ amino acid showed $60.2 \%$ sequence similarity with zebrafish and $41.1 \%$ sequence similarity with human and mouse, whereas conserved TIR domain of channel catfish identity ranged from $82.2 \%$ with zebrafish and $65.1 \%$ with flounder (Baoprasertkul et al., 2007). However, in our study, $m$ tlr 2 showed $58.1 \%$ amino acid sequence similarity with zebra fish $t l r 2$ while only 38.7 $\%$ amino acid sequence identity with human and $37.9 \%$ with mouse. TIR domain of $m t l r 2$ shares highest identity with other catfish group i.e. 89.9-87.64\%, 77.5\% with zebrafish, $64 \%$ with mouse and $65.2 \%$ with human. The conserved TIR domain of $m t l r 2$ identity ranged from $89.9 \%$ with pangasius to lowest $57.4 \%$ with Japanese flounder. This indicates huge structural variability in TIR domain between fresh water and marine water fishes.

Basal expression analysis of $m t l r 2$ and its downstream-signaling molecule $m y d 88, n f-k b$ and $i l-1 \beta$ showed constitutive expression in all the twelve tissues examined. The $m t l r 2$ showed higher level of basal expression in spleen and lower basal expression in skin. High level of $t l r 2$ basal expression has been detected in liver of channel catfish, spleen of yellow catfish, spleen of rohu, liver of turbot, muscle of tongue sole, head kidney of ray fish, blood of golden pompano, head-kidney of common carp. Lowest level of tlr2 basal expression has been detected in skin and muscle of channel catfish, eye of yellow cat fish, eye and skin of rohu, skin of turbot, intestine 
371 and blood of tongue sole, liver of golden pompano, muscle in common carp, intestine of ray fish,

372 (Baoprasertkul et al., 2007; Samanta et al., 2012; Liu et al., 2016; Li and Sun, 2016; Fink et al.,

373 2016; Zhang et al., 2017; Wu et al., 2018; Tang et al., 2020). The variation in the basal

374 expression of $t l r 2$ in different teleosts might be attributed to their wide diversity in habitat and

375 the microbial invaders they encounter. In the present study, highest basal expression of myd88

376 was detected in the spleen and lowest in the muscle. Basal expression analysis of myd88 in

377 Yellow catfish (Pelteobagrus fulvidraco) showed ubiquitous expression with the highest myd88

378 expression detected in spleen and the lowest in liver (Yu et al., 2018). The basal expression of $n f$ -

$379 k b$ in magur was lowest in muscle, and highest in anterior kidney. Literature survey shows that

380 till now there is no study on basal expression of this very important gene of TLR pathway in any

381 fish species. This is the first report on basal expression of $n f-k b$ gene in magur. Further, in this

382 study, lowest basal expression of $i l-1 \beta$ observed in muscle and calibrated higher basal expression

383 in spleen. In large yellow croaker $l c i l-1 \beta$ showed higher expression in head kidney followed by

384 gill, liver, and intestine ( $W u$ et al., 2015).

$385 t l r 2$ responds to various ligand (PGN, LTA, LPS, zymosan etc.) and pathogens in higher

386 vertebrates as well as in lower vertebrates like teleosts. Our finding also validates that $m t l r 2$ is

387 induced in response to $S$. aureus in several tissues of magur. As literature suggests that PGN is

388 the major cell wall component of this bacterium, which might be responsible for inducing

389 expression of $m t l r 2$. Inductive expression of $m t l r 2$ in response to PGN of Gram-positive bacteria

390 have been reported in large orange spotted grouper (Wei et al., 2011), yellow croaker (Fan et al.,

391 2015), rohu (Samanta et al., 2012), common carp (Ribeiro et al., 2010; Fink et al., 2016). There

392 are few reports on induction of fish tlr 2 to Gram-negative pathogens also. Significant up-

393 regulation of $l c t l r 2$ transcript in yellow croaker was detected after $V$. parahaemolyticus infection

394 and LPS stimulation (Fan et al., 2015). Up-regulated expression of $t l r 2$ in response to $A$.

395 hydrophila has been reported in channel catfish, hybrid sturgeons, yellow catfish (Mu et al.,

396 2013; Zhang et al., 2017; Wen et al., 2017; Jiang et al., 2018). This could be due to binding of

397 tlr2 to lipoproteins, PGN carried by A. hydrophila (Zhang et al., 2017). These findings suggest

398 that the $t l r 2$ in fish may also recognise Gram-negative bacteria in addition to its known ligands,

399 but more evidence is needed to substantiate this. .

$400 \quad$ Significant up-regulation of $m t l r 2$ detected in gill, liver, spleen, intestine and kidney at 3 ,

4018 or 24 hpi indicating early response of $t l r 2$ to $A$. hydrophila infection in magur was observed in 
402 our study. These findings are supported by Mu et al. (2013), they have reported that

403

404

405

406

407

408

409

410

411

412

413

414

415

416

417

418

419

420

421

422

423

424

425

426

427

428

429

430

431

transcriptional kinetics of the $\operatorname{tlr} 2$ peaked in the anterior kidney ( $\sim 5$ fold increase) of channel

catfish at $3 \mathrm{~h}$ post exposure to A. hydrophila. Zhang et al. (2017) also reported that A. hydrophila infection increased tlr 2 expression in spleen, head kidney and trunk kidney at 12 or 24 hpi. $A$. hydrophila infection challenge in mrigal induced tlr 2 expression in kidney ( 4.5 fold at $12 \mathrm{~h})$, gill ( $\sim 1.5$ fold at 12h) and in the liver, intestine (Basu et al., 2012). Our result shows that, as a part of innate immune defence system, $m$ tlr 2 expression is enhanced following $A$. hydrophila infection in magur.

In this study, a significant up-regulated $m t l r 2$, myd $88, n f-k b, i l-1 \beta$ gene expression observed in liver, spleen, intestine and kidney at 3, 8, 24 hpi, in gill at 8 or 24 hpi. myd88 is a key adopter molecule and play an important role in facilitating downstream signaling of all toll-like receptors except $t l r 3$. Our finding is supported by Huang et al. (2018), they reported a significant higher level of myd88 expression at 8 hpi in Anguilla japonica infected with E. tarda in head kidney, spleen, liver, intestine, gill and skin. In Wuchang bream (Megalobrama amblycephala), myd88 expression peaked at 12 hpi of A. hydrophila (Lai et al., 2017). All the TLR pathways culminate in activation of transcription factor $n f-k b$ which control expression of wide array of cytokines (Kawai and Akira, 2007)., The effecter cytokine molecule il-1 $\beta$ play crucial role in physiological events such as immune response, inflammation, cell growth and differentiation, apoptosis. Significantly up-regulated expression of $n f-k b$ and $i l-1 \beta$ in the spleen and muscle has been reported in koi carp following A. sobria infection (Byadgi et al., 2018).

A well-correlated expression pattern of $m t l r 2$ and the signaling molecules i.e. $m y d 88, n f$ $k b$ and $i l-1 \beta$ was observed in the respective tissues, during the initial hours (3 to 24 hpi) of infection. Higher expression of $m t l r 2, m y d 88, n f-k b, i l-1 \beta$ in internal organ such as intestine, kidney, spleen and liver than gill may be due to intra-peritoneal mode of experimental bacterial infection. Further, it also can be speculated that intestine and kidney are the two earliest responding organs to A. hydrophila infection. The results obtained in the present study are in agreement with the findings by Samanta et al. (2012) and Li and Sun (2016), on regulation of tlr2 and its downstream-signaling molecules in rohu and tongue sole, respectively following bacterial infections.

\section{Conclusion}


In conclusion, full-length cDNA sequence of magur $t l r 2$ was generated and characterised.

433 A well-correlated expression pattern of $m t l r 2$ and the downstream signaling molecules i.e. $m y d 88, n f-k b$ and $i l-1 \beta$ was observed in gill, liver, spleen, intestine and kidney during the initial hours (3 to 24 hpi) of $A$. hydrophila infection in magur cat fish.

\section{Abbreviation list}

PRR- pathogen recognition receptor (PRR), tlr-toll-like receptor, myd88- myeloid differentiation primary response 88, cDNA- complimentary DNA, LRR- leucine-rich repeat region, TIR- tollinterleukin 1 ( $i l-1)$ receptor, PGN- peptidoglycan, LTA- lipoteichoic acid, $n f-k b$ - nuclear Factor kappa-light-chain-enhancer of activated $\mathrm{B}, i l-1 \beta$ - interleukin 1 beta, PAMP-pathogen-associated molecular pattern, DAMP- damage-associated molecular pattern, LPS- lipopolysaccharides, ORF- open reading frame, pi- isoelectic point, TSB- tryptic-soy broth, DEPC- diethyl pyrocarbonate.

\section{Acknowledgements}

The authors wish to express their sincere thanks to Dr. Kuldeep K. Lal, Director, ICAR-NBFGR, Lucknow, India for providing the facilities and his support, guidance and encouragement. The authors would also like to sincerely acknowledge the reviewers for their constructive remarks which have helped to improve the manuscript.

\section{Funding}

This work was carried out with the financial in-house funding support under the project code FISHNBFGRSIL201600800189.

\section{Conflict of interest}

457 It is declared by authors that there is no conflict of interest that could influence the work reported

458 in this paper and all others have consented for the publication and authorship. 459

\section{Data availability statement}

461 All the data supporting the findings of this study are available within the article and raw data is 462 available as supplementary file. 


\section{Author's contribution}

465

466

467

468

469

470

471

472

473

474

475

476

477

478

479

480

481

482

483

484

485

486

487

488

489

490

491

492

493

Chinmayee Muduli: Data curation, writing original draft, Anutosh Paria: Review and editing,

Ranjana Srivastava: Data curation, Gaurav Rathore: Conceptualization, Review and editing, Methodology, Supervision, Kuldeep K Lal: Conceptualization, Methodology, Supervision. All authors have gone through the final version of the manuscript.

\section{Ethics approval}

a) The animal care and experimental challenge was approved by the Institutional Animal Ethics Committee of ICAR-NBFGR, Lucknow.

b) The article does not contain any studies involving human participants performed by any of the authors.

\section{References}

1. Akira S, Uematsu S, Takeuchi O. 2006. Pathogen recognition and innate immunity. Cell 124(4):783-801. DOI 10.1016/j.cell.2006.02.015

2. Anandhakumar C, Lavanya V, Pradheepa G, Tirumurugaan KG, Raj DG, Raja A, Pazhanivel N, Balachandran C. 2012. Expression profile of toll-like receptor 2 mRNA in selected tissues of shark (Chiloscyllium sp.). Fish and Shellfish Immunology 33(5):1174-1182. doi: 10.1016/j.fsi.2012.09.007

3. Baoprasertkul P, Peatman E, Abernathy J, Liu Z. 2007. Structural characterisation and expression analysis of Toll-like receptor 2 gene from catfish. Fish and Shellfish Immunology 22:418-426. doi:10.1016/j.fsi.2006.04.005

4. Basu M, Swain B, Sahoo BR, Maiti NK, Samanta M. 2012. Induction of toll-like receptor (TLR) 2, and MyD88-dependent TLR- signaling in response to ligand stimulation and bacterial infections in the Indian major carp, mrigal (Cirrhinus mrigala). Molecular Biology Reports 39:6015-6028. DOI: 10.1007/s11033-011-1415-9

5. Brietzke A, Arnemo M, Gjøen T, Rebl H, Korytar T, Goldammer T, Rebl A, Seyfert HM. 2016. Structurally diverse genes encode Tlr2 in rainbow trout: The conserved receptor cannot be stimulated by classical ligands to activate NF- $\kappa \mathrm{B}$ in vitro. Developmental and Comparative Immunology 54(1):75-88. doi:10.1016/j.dci.2015.08.012 
494

495

496

497

498

499

500

501

502

503

504

505

506

507

508

509

510

511

512

513

514

515

516

517

518

519

520

521

522

523

524

6. Bustin SA, Benes V, Garson JA, Hellemans J, Huggett J, Kubista M, Mueller R, Nolan T, Pfaffl MW, Shipley GL. 2009. The MIQE guidelines: minimum information for publication of quantitative real-time PCR experiments. Clinical Chemistry 55(4):611622. 10.1373/clinchem.2008.112797.

7. Buwittbeckmann U, Heine H, Wiesmüller KH, Jung G, Brock R, Akira S, Ulmer AJ. 2006. TLR1- and TLR6-independent recognition of bacterial lipopeptides. Journal of Biological Chemistry 281:9049-9057. doi:10.1074/jbc.M512525200

8. Byadgi O, Chen YC, Maekawa S, Wang PC, Chen SC. 2018. Immune-related functional differential gene expression in koi carp (Cyprinus carpio) after Challenge with Aeromonas sobria. International Journal of Molecular Sciences 19(7):2107. doi: $10.3390 /$ ijms 19072107

9. Chena Y, Lub S, Zhang Y, Yud J, Denga L, Chena H, Zhang Y, Zhou N, Yuan K, Yua L, Xionga Z, Guia X, Yu Y, Min W. 2019. TLR2 agonist Pam3CSK4 enhances the antibacterial functions of GM-CSF induced neutrophils to methicillin-resistant Staphylococcus aureus. Microbial Pathogenesis 130:204-212. doi:10.1016/j.micpath.2019.02.030

10. Devassy A, Kumar R, Shajitha PP, John R, Padmakumar KG, Basheer VS, Gopalakrishnan A, Mathew L. 2015. Genetic identification and phylogenetic relationships of Indian clariids based on mitochondrial COI sequences. Mitochondrial DNA 1:4. DOI: $\underline{10.3109 / 19401736.2015 .1079901}$

11. Fan ZJ, Jia QJ, Yao CL. 2015. Characterization and expression analysis of toll-like receptor 2 gene in large yellow croaker, Larimichthys crocea. Fish and Shellfish Immunology 44:129-137. DOI: 10.1016/j.fsi.2015.01.037

12. Fink IR, Pietretti D, Voodgt CGP, Westphal AH, Savelkoul HFJ, Forlenza M, Wiegertjes GF. 2016. Molecular and functional characterization of Toll-like receptor (TLR)1 and TLR2 in common carp (Cyprinus carpio). Fish and Shellfish Immunology 56:70-83. doi:10.1016/j.fsi.2016.06.049

13. Gao Q, Xiao Y, Zhang C, Min M, Peng S, Shi Z. 2016. Molecular Characterization and Expression Analysis of Toll-like Receptor 2 in Response to Bacteria in Silvery Pomfret Intestinal Epithelial Cells. Fish and Shellfish Immunology 58:1-9. DOI: 
525

526

527

528

529

530

531

532

533

534

535

536

537

538

539

540

541

542

543

544

545

546

547

548

549

550

551

552

553

554

555

14. He LB, Wang H, Luo LF, Jiang SH, Liu LY, Li YM, Huang R, Liao LJ, Zhu ZY, Wang YP. 2016. Characterization, expression analysis and localization pattern of toll-like receptor $1(\mathrm{t} l \mathrm{r} 1)$ and toll-like receptor $2(\mathrm{t} l \mathrm{r} 2)$ genes in grass carp Ctenopharyngodon idella. Journal of Fish Biology 89:1434-1440. doi:10.1111/jfb.12997

15. Hikima J, Jung TS, Aoki T. 2011. Immunoglobulin genes and their transcriptional control in teleosts. Developmental and Comparative Immunology 35:924-936. doi:10.1016/j.dci.2010.10.011.

16. Hirono I, Takami M, Miyata M, Miyazaki T, Han HJ, Takano T, Endo M, Aoki T. 2004. Characterization of gene structure and expression of two toll-like receptors from Japanese flounder, Paralichthys olivaceus. Immunogenetics 56:38-46. doi:10.1007/s00251-0040657-2

17. Huang WS, Wang ZX, Liang Y, Nie P, Huang B. 2018. Characterization of MyD88 in Japanese eel, Anguilla japonica. Fish and Shellfish Immunology 81:374-382. doi:10.1016/j.fsi.2018.07.028

18. Jault C, Pichon L, Chluba J. 2004. Toll-like receptor gene family and TIR-domain adapters in Danio rerio. Molecular Immunology 40(11):759-771. DOI: 10.1016/j.molimm.2003.10.001

19. Jiang N, Fan Y, Zhou Y et al. 2018. Transcriptome analysis of Aeromonas hydrophila infected hybrid sturgeon (Huso dauricus $\times$ Acipenser schrenckii). Scientific Reports 8:17925.

20. Kawai T, Akira S. 2007. Signaling to NF-kappaB by Toll-like receptors. Trends in Molecular Medicine 13(11):460-469. doi:10.1016/j.molmed.2007.09.002.

21. Kawai T, Akira S. 2010. The role of pattern-recognition receptors in innate immunity: update on Toll-like receptors. Nature Immunology 11(5):373-384.

DOIhttps://doi.org/10.1038/ni.1863

22. Kubista M, Andrade JM, Bengtsson M, Forootan A, Jonak J, Lind K. 2006. The realtime polymerase chain reaction. Molecular Aspects of Medicine 27:95-125.

23. Lai RF, Jakovlić I, Liu H, Wei J, Zhan FB, Yang PH, Wang WM. 2017. Characterization and expression of Megalobrama amblycephala toll-like receptor 22 involved in the response to Aeromonas hydrophila. Journal of Fish Biology 90(3):803-818. doi:10.1111/jfb.13199.

Peer] reviewing PDF | (2021:04:59932:2:0:NEW 19 Sep 2021) 
556

557

558

559

560

561

562

563

564

565

566

567

568

569

570

571

572

573

574

575

576

577

578

579

580

581

582

583

584

585

24. Li XP, Sun L. 2016. Toll-like receptor 2 of tongue sole Cynoglossus semilaevis: Signaling pathway and involvement in bacterial infection. Fish and Shellfish Immunology 51:321-328. doi:10.1016/j.fsi.2016.03.001

25. Liao Z, Wan Q, Su H, Wu C, Su J. 2017. Pattern recognition receptors in grass carp Ctenopharyngodon idella: I. Organization and expression analysis of TLRs and RLRs. Developmental and Comparative Immunology 76:93-104.

DOI: 10.1016/j.dci.2017.05.019

26. Liu F, Su B, Gao C, Zhou S, Song L, Tan F, Dong X, Ren Y, Li C. 2016. Identification and expression analysis of TLR2 in mucosal tissues of turbot (Scophthalmus maximus L.) following bacterial challenge. Fish and Shellfish Immunology 55:654-661. doi:10.1016/j.fsi.2016.06.047

27. Liu Y, Wang Y, Yamakuchi M, Isowaki S, Nagata E, Kanmura Y, Kitajima I, Maruyama I. 2001. Upregulation of Toll-like Receptor 2 gene expression in macrophage response to

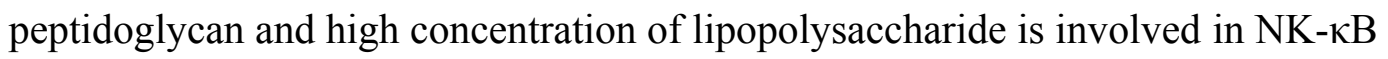
activation. Infection and Immunity 69(5):2788-96. doi: 10.1128/IAI.69.5.2788$\underline{2796.2001}$

28. Livak KJ, Schmittgen TD. 2001. Analysis of relative gene expression data using realtime quantitative PCR and the method. Methods 25:402-408. doi:10.1006/meth.2001.1262

29. Matsushima N, Tanaka T, Enkhbayar P, Mikami T, Taga M, Yamada K, Kuroki Y. 2007. Comparative sequence analysis of leucine-rich repeats (LRRs) within vertebrate toll-like receptors. BMC Genomics 8:124. doi: 10.1186/1471-2164-8-124

30. Mogensen TH. 2009. Pathogen recognition and inflammatory signaling in innate immune defenses. Clinical Microbiology Reviews 22(2):240-73. doi: 10.1128/CMR.00046-08

31. Mogensen TH, Paludan SR. 2005. Reading the viral signature by toll-like receptors and other pattern recognition receptors. Journal of Molecular Medicine 83:80-92. doi:10.1007/s00109-004-0620-6

32. Monir MS, Bagum N, LutfulKabir SM, Chakraborty S, Ashaf M, UdDoulah MA. 2017. Isolation, molecular identification and characterization of Aeromonas hydrophila from infected air-breathing catfish Magur (Clarias batrachus) cultured in Mymensingh. Bangladesh Asian-Australasian Journal of Food Safety and Security 1 (1):17-24. 
586

587

588

589

590

591

592

593

594

595

596

597

598

599

600

601

602

603

604

605

606

607

608

609

610

611

612

613

614

615

33. Mu X, Pridgeon JW, Klesius PH. 2013. Comparative transcriptional analysis reveals distinct expression patterns of channel catfish genes after the first infection and reinfection with Aeromonas hydrophila. Fish and Shellfish Immunology 35(5):1566-1576. doi:10.1016/j.fsi.2013.08.027

34. Muduli C, Tripathi G, Prasad KP, Kumar K, Singh RK, Rathore G. 2020. Virulence potential of Aeromonas hydrophila isolated from apparently healthy freshwater food fish. Biologia 76(3):1005-15. DOI 10.2478/s11756-020-00639-z.

35. O’Mahony DS, Pham U, Iyer R, Hawn TR, Liles WC. 2008. Differential constitutive and cytokine-modulated expression of human Toll-like receptors in primary neutrophils, monocytes, and macrophages. International Journal of Medical Sciences 5:1-8. doi: $\underline{10.7150 / \mathrm{ijms} .5 .1}$

36. Oshiumi H, Tsujita T, Shida K, Matsumoto M, Ikeo K, Seya T. 2003. Prediction of the prototype of the human Toll-like receptor gene family from the pufferfish, Fugu rubripes, genome. Immunogenetics 54(11):791-800. doi:10.1007/s00251-002-0519-8

37. Ozinsky A, Underhill DM, Fontenot JD, Hajjar AM, Smith KD, Wilson CB, Schroeder L, Aderem A. 2000. The repertoire for pattern recognition of pathogens by the innate immune system is defined by cooperation between Toll-like receptors. Proceedings of the National Academy of Sciences of the United States of America 97:13766-13771. https://doi.org/10.1073/pnas.250476497

38. Ribeiro CMS, Hermsen GJ, Taverne-Thiele JJ, Savelkoul HFJ, Wiegertjes GF. 2010. Evolution of recognition of ligands from gram-positive bacteria: similarities and differences in the TLR2-mediated response between mammalian vertebrates and teleost fish. The Journal of Immunology 184(5):2355-68. doi:10.4049/jimmunol.0900990

39. Sahoo SK, Ferosekhan S, Giri SS, Swain SK. 2016. Recent trends in breeding and seed production of magur in India. World Aquaculture 47(2):59-62.

40. Samanta M, Swain B, Basu M, Panda P, Mohapatra GB, Sahoo BR, Maiti NK. 2012. Molecular characterization of toll-like receptor 2 (TLR2), analysis of its inductive expression and associated down-stream signaling molecules following ligands exposure and bacterial infection in the Indian major carp, rohu (Labeo rohita). Fish and Shellfish Immunology 32:411-425. doi:10.1016/j.fsi.2011.11.029 
616

617

618

619

620

621

622

623

624

625

626

627

628

629

630

631

632

633

634

635

636

637

638

639

640

641

642

643

644

645

646

41. Sharma A, Chanu TI, Ande MP, Jahageerdar S. 2018. Common diseases and aberration in walking Catfish (Clarias magur, Hamilton, 1822) and related Advisories. Aquaculture times 4(4).

42. Sharma A, Chanu TI, Ande MP, SureshBabu PP, Syamala K, Rao PS, Deo AD, Jahageerdar S. 2017. Virulent Aeromonas veronii Strain BLB-01 associated with mass mortality of Clarias batrachus (Linnaeus, 1758). International Journal of Current Microbiology and Applied Sciences 6(8):3668-3681. DOI: 10.20546/ijcmas.2017.608.444

43. Sinha M, Mahapatra BK, Saha D, Maitra NJ. 2014. Mass scale seed production of Magur, Clarias batrachus at farm level through improvised modifications. International Journal of Fisheries and Aquatic Studies 2(2):210-214.

44. Takeuchi O, Akira S. 2010. Pattern Recognition Receptors and Inflammation. Cell 140(6):805-820. doi:10.1016/j.cell.2010.01.022

45. Takeuchi O, Kawai T, Muhlradt PF, Morr M, Radolf JD, Zychlinsky A, Takeda K, Akira S. 2001. Discrimination of bacterial lipoproteins by Tolllike receptor 6. International Immunology 13:933-940. doi:10.1093/intimm/13.7.933

46. Takeuchi O, Sato S, Horiuchi T, Hoshino K, Takeda K, Dong Z, Modlin RL, Akira S. 2002. Cutting and expression analysis of toll-like receptor 2 gene from catfish. Fish and Shellfish Immunology 22:418-426.

47. Tang R, Wang S, Han P, Zhang Q, Zhang S, Xing X, Shao R, Xu W, Xu Q, Wei Q, Qi Z. 2020. Toll-like receptor (TLR) 2 and TLR13 from the endangered primitive-ray finned fish Dabry's sturgeon (Acipenser dabryanus) and their expression profiling upon immune stimulation. Aquaculture Reports 16:100247. DOI: 10.1016/j.aqrep.2019.100247

48. Varriale S, Ferraresso S, Giacomelli S, Coscia MR, Bargelloni L, Oreste U. 2012. Evolutionary analysis of Antarctic teleost Toll-like receptor 2. Fish and Shellfish Immunology 33(5):1076-1085. doi:10.1016/j.fsi.2012.07.010

49. Wei YC, Pan TS, Chang MX, Huang B, Xu Z, Luo TR, Nie P. 2011. Cloning and expression of Toll-like receptors 1 and 2 from a teleost fish, the orange-spotted grouper Epinephelus coioides. Veterinary Immunology and Immunopathology 141(3-4):173-182. doi:10.1016/j.vetimm.2011.02.016

50. Wen X, Wang L, Zhu W, Wang D, Li X, Qian X, Yin S. 2017. Three toll-like receptors (TLRs) respond to Aeromonas hydrophila or lipopolysaccharide challenge in pufferfish, 
647

648

649

650

651

652

653

654

655

656

657

658

659

660

661

662

663

664

665

666

667

668

669

670

671

672

673

674

675

Takifugu fasciatus. Aquaculture 481(40-47).

https://doi.org/10.1016/j.aquaculture.2017.08.020.

51. Wu J, Shi YH, Zhang XH, Li CH, Li MY, Chen J. 2015. Molecular characterization of an IL-1 $\beta$ gene from the large yellow croaker (Larimichthys crocea) and its effect on fish defense against Vibrio alginolyticus infection. Zoological Research 36 (3):133- 141.

52. Wu M, Guo L, Zhu KC, Guo HY, Liu B, Jiang SG, Zhang DC. 2018. Genomic structure and molecular characterization of Toll-like receptors 1 and 2 from golden pompano Trachinotus ovatus (Linnaeus, 1758) and their expression response to three types of pathogen-associated molecular patterns. Developmental and Comparative Immunology 86:34-40. doi:10.1016/j.dci.2018.04.022

53. Xu T, Meng F, Zhu Z, Wang R. 2013. Characterization and comprehensive analysis of the miiuy croaker TLR2 reveals a direct evidence for intron insert and loss. Fish and Shellfish Immunology 34(1):119-128.

54. Yu L, Zhang L, Yang H, Gui G, Liu Y, Xiao Y. 2018. Identification and characterization of the myeloid differentiation factor 88 gene in yellow catfish. 3 Biotech 8:430. doi:10.1007/s13205-018-1448-z

55. Zhang H, Hu G, Liu Q, Zhang S. 2016. Cloning and expression study of a Toll-like receptor 2 (tlr2) gene from turbot, Scophthalmus maximus. Fish and Shellfish Immunology 59:137-148. doi:10.1016/j.fsi.2016.10.001

56. Zhang J, Kong X, Zhou C, Li L, Nie G, Li X. 2014. Toll-like receptor recognition of bacteria in fish: ligand specificity and signal pathways. Fish and Shellfish Immunology 41:380-388. DOI: 10.1016/j.fsi.2014.09.022

57. Zhang XT, Zhang GR, Shi ZC, Yuan YJ, Zheng H, Lin L, Wei KJ, Ji W. 2017. Expression analysis of nine Toll-like receptors in yellow catfish (Pelteobagrus fulvidraco) responding to Aeromonas hydrophila challenge. Fish and Shellfish Immunology 63:384-393. doi:10.1016/j.fsi.2017.02.021

58. Zhu LY, Nie L, Zhu G, Xiang LX, Shao JZ. 2013. Advances in research of fish immune relevant genes: a comparative overview of innate and adaptive immunity in teleosts. Developmental and Comparative Immunology 39: 39-62. doi:10.1016/j.dci.2012.04.001. 


\section{Figure 1}

Nucleotide and deduced amino acid sequence of $m t / r 2$ cDNA.

Start codon and stop codon are marked in magenta colour. In the deduced amino acid sequence, signal peptide (1-42 aa) is highlighted by magenta colour. LRR-NT and LRR-CT are highlighted in grey, LRRs are highlighted in yellow, transmembrane domain highlighted in turquoise and the cytoplasmic TIR domain is highlighted in olive green. Extracellular LRR-NT (50-73 aa), LRR1 (98-115 aa), LRR2 (122-139 aa), LRR3 (143-164 aa), LRR4 (173-187 aa), LRR5 (173-459 aa), LRR6 (197-215 aa), LRR7 (228-239 aa), LRR8 (250-268 aa), LRR9 (277-296 aa), LRR10 (299-316 aa), LRR11(331-347 aa), LRR12 (360-373 aa), LRR13 (387-401 aa), LRR14 (415-434 aa), LRR15 (439-456 aа), LRR16 (462-478 aa), LRR17 (483-498 aа), LRR18 (503-519 aa), LRR19 (525-535 aa), LRR20 (543-559 aa), LRR-CT (588-608 aa). Transmembrane domain (609-631aa), intracellular cytoplasmic TIR (670-783 aa). Two mRNA instability motifs (ATTTA) marked in red colour underlining. 
1 ATAGTTAAGCGAGAAAAGATTACTACAAGCAAGACTTTTATCTACTGGTGTACTTGATAATAACTACCGGTTTTGTTTTGTTTTGTTTTGTT92 93 TTTAGTTTTGATTCTACTGTGATGCAAATGCGACACTTCAGGCTGATGTTTTCAGCTTCTCTCCAGAAACAACACACATCCAGCAGGATG182 $\begin{array}{llllllllllllllllllllllll}M & \& & M & R & H & F & R & L & M & F & S & A & S & L & Q & K & Q & H & T & S & S & R & M\end{array}$

183AAGGTGCCACTGGCCTTATGTATCTGTTTTAGCCTGACTCTGACTCTGACTCAGACCTCCGAGAGACCAACCTGTAATGACTGCGACGAA2 72 $\begin{array}{lllllllllllllllllllllllllllllll}K & V & P & I & A & L & C & I & C & F & S & L & T & L & T & L & T & Q & T & S & E & R & P & T & C & N & D & C & D & E\end{array}$ 273GATCATTTCTGCAACTGTCGTGCTAAGGACCTCCACGGTGTCCCCATAGTTCCAGATGATGTTCTTTACCTAGACGTGTCCTTCAACGAG362 $\begin{array}{lllllllllllllllllllllllllllllllll}\text { D } & \text { H } & \text { F } & \text { C } & \text { N } & \text { C } & \text { R } & \text { A } & \text { K } & \text { D } & \text { L } & \text { H } & G & \text { V } & \text { P } & \text { I } & \text { V } & \text { P } & \text { D } & \text { D } & \text { V } & \text { L } & \text { Y } & \text { L } & \text { D } & \text { V } & \text { S } & \text { F } & \text { N } & E\end{array}$ 363ATCGAGTCCATCACTCAGAGGGATCTGACCTGCTACACAGAGCTGAGAAATTTAAAGCTGCAGAAGAACAAACTCAGCACGATCCACAAA452

$\begin{array}{llllllllllllllllllllllllllllllll}I & E & S & I & T & Q & R & D & L & T & C & Y & T & E & L & R & N & L & K & L & Q & K & N & K & L & S & T & I & H & K\end{array}$ 453GAAGCATTTCATTCCCAAAGTAAACTGGAAGCGCTTGATCTGTCATTCAATAACCTGAAAAACATTTCCTCCCAATGGTTTTCTAATCTT542 $\begin{array}{llllllllllllllllllllllllllllllllllll} & E & A & F & H & S & Q & S & K & L & E & A & L & D & L & S & F & N & N & L & K & N & I & S & S & Q & W & F & S & N & L\end{array}$ 543CGGTCCCTGAAACATTTGAACATCTTGGGAAACCAGTACACCACTTTGGGATCCATCGCCTTGTTTCAATTTGTCGAAAACCCCGCGCTG632

$\begin{array}{llllllllllllllllllllllllllllllllll}R & S & \text { L } & \text { K } & \text { H } & \text { L } & \text { N } & \text { I } & \text { L } & G & \text { N } & \text { Q } & \text { Y } & \text { T } & \text { T } & \text { L } & G & \text { S } & \text { I } & \text { A } & \text { L } & \text { F } & \text { Q } & \text { F } & \text { V } & \text { E } & \text { N } & \text { P } & \text { A } & \text { L }\end{array}$ 633AGAACGTTACAGTTCGGCAACCTTTGGATCAGGGATGTGAAACAGAATTTGCTGCGTAATATTAGACAGCTGGATGAGCTGTCGTTTGTC722

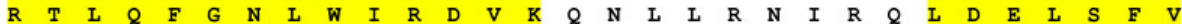
723GGTGGTGTCCTCAGATCATATGAGAATGGAAGCTTCCAGACGATTCAACCCATCAGAGCCGTGTCAGTCAGCCTTTCGCGGTTGTTTCAG812 $\begin{array}{llllllllllllllllllllllllllllll}G & G & V & L & R & S & Y & E & N & G & S & F & Q & T & I & Q & P & I & R & A & V & S & V & S & L & S & R & L & F & Q\end{array}$ 813GATGATCCAGCACTGGTATCAAAGATCCTTCGAGATGTTTCTCACCCTGAGACATCGCTGACCATTAGAGATGTCTCCCTGGAGACACAA902 $\begin{array}{llllllllllllllllllllllllllllllll}\text { D } & \text { D } & \text { P } & \text { A } & \text { L } & \text { V } & \text { S } & \text { K } & \text { I } & \text { L } & \text { R } & \text { D } & \text { V } & \text { S } & \text { H } & \text { P } & \text { E } & \text { T } & \text { S } & \text { L } & \text { T } & \text { I } & \text { R } & \text { D } & \text { V } & \text { S } & \text { L } & E & T & Q\end{array}$ 903GAACTGATAGAACCCTTAAGAGAGGTGACAGAAGGTGGCACCAGAAGTCTTACCTTTCAAAACATAATCACAACTGACGAGGCAGTCAGC992

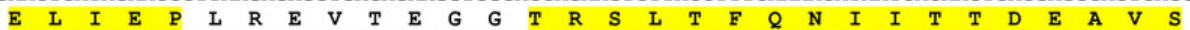
993CGCCTTCTGGAGGTTTTGGACGGCTCTCCGGTGTCCTACATCGGCCTTGAGGACATTTGTTTAATAGGTCAGGGCTGGTGGGAAAAGGCG1082

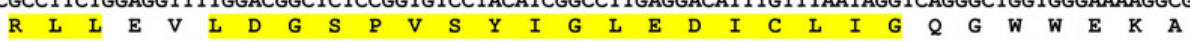
1083AAGAGGACACACCTAGAAAACCTGCACACGATACATGTCCGCAACATAGAAATCCAGGGCTTCTTCAAATTTAGCAGCATGATACAGTTA1172

$\begin{array}{lllllllllllllllllllllllllllllllll}K & R & T & H & \text { L } & E & \text { N } & \text { L } & H & \text { T } & \text { I } & H & \text { V } & R & \text { N } & \text { I } & E & \text { I } & Q & G & F & F & K & F & S & S & M & I & Q & L\end{array}$ 1173GCGTTCCTGTTGAAGCACCTCACCAAGATATCCGTCATCAACTGCACCGTTTTCGTTATTCCCTGCCTGACCAGCTGTTTTCTTAAAAAG1262 $\begin{array}{llllllllllllllllllllllllllllllll}\text { A } & F & \text { L } & \text { L } & K & \text { H } & \text { L } & \text { T } & K & \text { I } & \text { S } & \text { V } & \text { I } & \text { N } & \text { C } & \text { T } & \text { V } & \text { F } & \text { V } & \text { I } & \text { P } & \text { C } & \text { L } & \text { T } & \text { S } & \text { C } & \text { F } & \text { L } & K & K\end{array}$ 1263GTGGAGTACTTGGACTTGAGCCAAAACCTCCTCTCGGATATCACCATGCAAGAATCCCTGTGCAACGGGGACAGCAAGATGCGCAATATT1352 $\begin{array}{lllllllllllllllllllllllllllllllll}V & E & Y & L & D & L & S & O & N & L & L & S & D & I & T & M & O & E & S & L & C & N & G & D & S & K & M & R & N & I\end{array}$ 1353AACACGCTCAATGTAAGTCACAACTCGCTGAAATCTCTGCAGCTCATGTCCCACCTGGTCACGAGTCTCGACAGGCTGACATCGCTAGAC1442 $\begin{array}{llllllllllllllllllllllllllllllll}\text { N } & \text { T } & \text { L } & \text { N } & \text { V } & \text { S } & \text { H } & \text { N } & \text { S } & \text { L } & \text { K } & \text { S } & \text { L } & \text { Q } & \text { L } & \text { M } & \text { S } & \text { H } & \text { L } & \text { V } & \text { T } & \text { S } & \text { L } & \text { D } & \text { R } & \text { L } & \text { T } & \text { S } & \text { L } & \text { D }\end{array}$ 1443ATGAGCCACAACAACTTTGTAAAGATGCCACAGAGTTGCAGCTGGCCGGCAAGTCTCAGGTTTATGAACCTGTCCACTACAAAACTTCAC1532 $\begin{array}{lllllllllllllllllllllllllllllllllll}M & S & H & N & N & F & V & K & M & P & Q & S & C & S & \text { W } & \text { P } & \text { A } & \text { S } & \text { L } & \text { R } & \text { F } & \text { M } & \text { N } & \text { L } & \text { S } & \text { T } & \text { T } & \text { K } & \text { L } & \text { H }\end{array}$ 1533CGCGTAACCCCGTGCCTACCTCTCAGCCTGACCGTGCTGGATTTGAGCCAGAACTTCCTGACAGAGTTCCACCTCCATCTTCCCAACCTT1662

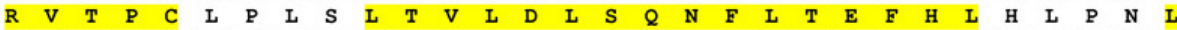
1663GCGGAGCTCTGGCTTACAGGGAACAGGATTATTGCCCTGCCGGAAGGTGGCCACTTCCCCAGCCTACGCATGCTGTTTATTCAAAGCAAC1712 $\begin{array}{lllllllllllllllllllllllllllllllllllll}A & E & L & \text { W } & L & T & G & N & R & I & I & A & L & P & E & G & G & H & F & P & S & L & R & M & L & F & I & Q & S & N\end{array}$ 1713ACATTGAACATGTTCAACAAAAGCGACCTGATGGCGTTCCAGTCTCTCCAGGTCTTGGAAGCCGGACATAACAATTTTTTTTGCAGCTGC1802 $\begin{array}{lllllllllllllllllllllllllllllll}T & \text { L } & \text { N } & M & \text { F } & \text { N } & \text { K } & \text { S } & \text { D } & \text { L } & \text { M } & \text { A } & \text { F } & \text { Q } & \text { S } & \text { L } & \text { Q } & \text { V } & \text { L } & \text { E } & \text { A } & G & \text { H } & \text { N } & \text { N } & \text { F } & \text { F } & \text { C } & \text { S } & \text { C }\end{array}$ 1803GATTTCGTAGAATTCTTTCAAGGTTCTATTGACCACTTGATCACTCTGGGGGACGGACATCGCAGCTACATGTGTGACTCTCCGTTCACG1892

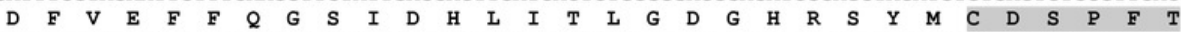
1893TTAAGGGGTCTTAATATAGATACCGCTCAACCGCCAGTCTTCGAGTGCTACATGATCCTGTTAGTATCAGTCATCTGCTCGGTCACCGTC1982 $\begin{array}{lllllllllllllllllllllllllllllllllll}L & R & G & L & N & I & D & T & A & Q & P & P & V & F & E & C & Y & M & I & L & L & V & S & V & I & C & S & V & T & V\end{array}$ 1983ATCGGCGTGATCGCCATCGGGTCACCTGCCACAAATTCCACATCTTGTGGTACCTGCAGATGATGATCGCGTGGTTAAAAGCAAAGAGT2072

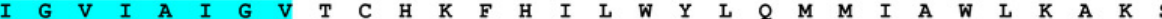
2073AAACCATCCGTGCAAATGGCGGCGTACTTTTCGATACGATGCTTTTCGTGTCGTACAGCCAGCACGATGCGCAGTGGGTGGAGGAAATC2162

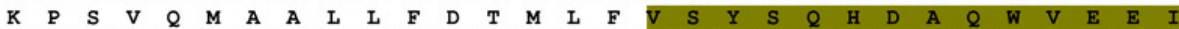
2163CTCGTGCCAGAGTTAAAAAGCTCTGAGTCTCCGCTCGCTCTGTGTCTGCACCAGCGGGACTTCCTCCCAGGCCGCTGGATCGCCGACAAC2252

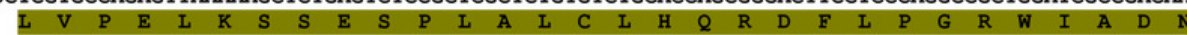
2253ATCATCGAGTCCATCGAAAGCAGCTATCGGACCCTCTTTGTCCTGTCGGAGAACTTCGTGACGAGCGAGTGGTGCCGATACGAGCTGAAC2342 $\begin{array}{lllllllllllllllllllllllllllllll} & I & I & E & S & I & E & S & S & Y & R & T & L & F & V & L & S & E & N & F & V & T & S & E & W & C & R & Y & E & L & N\end{array}$ 2343TTTTCGCATTTTCGGATCATCGACGAGCGCAACGATTCGGCGTCCCTGATCCTGCTAGAGCCCATCGCCAAGGAGACGATTCCCAAGCG2432

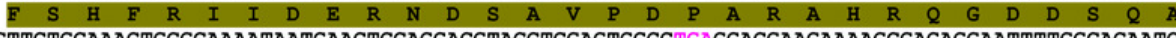
2433CTTCTGCAAACTGCGCAAAATAATGAACTCCAGGACGTACCTCGAGTGGCCTGAGGACGAAGAAAAGCGAGAGGAATTTTGGCACAATCT2522 $\begin{array}{lllllllllllllllllllll}L & L & Q & T & A & Q & N & N & E & L & Q & D & V & P & R & V & A & -\end{array}$

2523CCGAGCTGCACTTAGAAGGGAGGACTCGTGATGCCGATTCTGATACGTCACGGATCACACCTTCATCAGATTTGAAAATAATTACCCGGT2612 2613TTCATTTTGTCAGTAATTTGAAGTTCTGACAGCGGAGAACCCTTACTTGTTACTTGTGGTGTGTCTACAGCATACGCACTTATTTACCGT2702 2703TAGAGCTCGTTATTCCTCACCTGATGTACAGATTAGACTCAAACCCAGACTCAACCTCTGATTAAGTTCTGCTCTCAATAAACACAAGCT2792 2793CTATCTATTTAAGGATCTTCTGCATCGGGGGCTTTCCTTTTTATGGTTAGCTTCCTCATAAAATGGAGAACACACACACACACACATGCA2882 2883CACATCTTATGAGTAATTCTTCAGTTATTCACTCAATGAAACCTTCCTCATGATGGTTAGATTCGTGCTGTCTTGTTTTAATATGATTTT2972 2973ATATTACAGAAGTGGAAAGATTATTAATAATTAACTTGTGAAAATAAACGCAGCGAGGAATCATTTCAAAAAAAAAAAAAAAAAAAAAAA3062 3063AAAA 
Figure 2

Schematic representation of $C$. magur t/r2 (mt/r2) protein domains.

Signal peptide (pink), LRR domains (yellow), LRR-CT and LRR-NT (grey) followed by transmembrane and TIR domain (blue). 


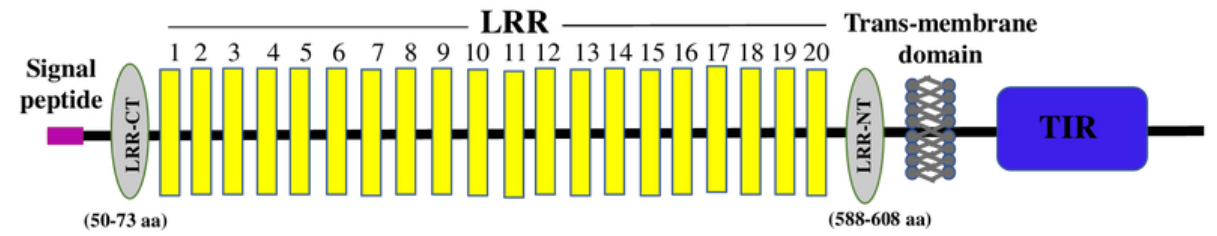




\section{Figure 3}

Alignment of $t / r 2$ protein sequences.

The sequences from human, zebra fish, rohu, common carp, large yellow croaker and channel catfish were aligned with magur t/r2 sequence to identify the conserved features and domains. Dashes represented amino acid deletions. The signal peptide is underlined, LRRs are highlighted in grey, transmembrane domain is indicated by boxes and also highlighted in grey, and the TIR domain is highlighted in black. Furthermore, LRRNT and LRRCT cysteines are indicated with arrows. 


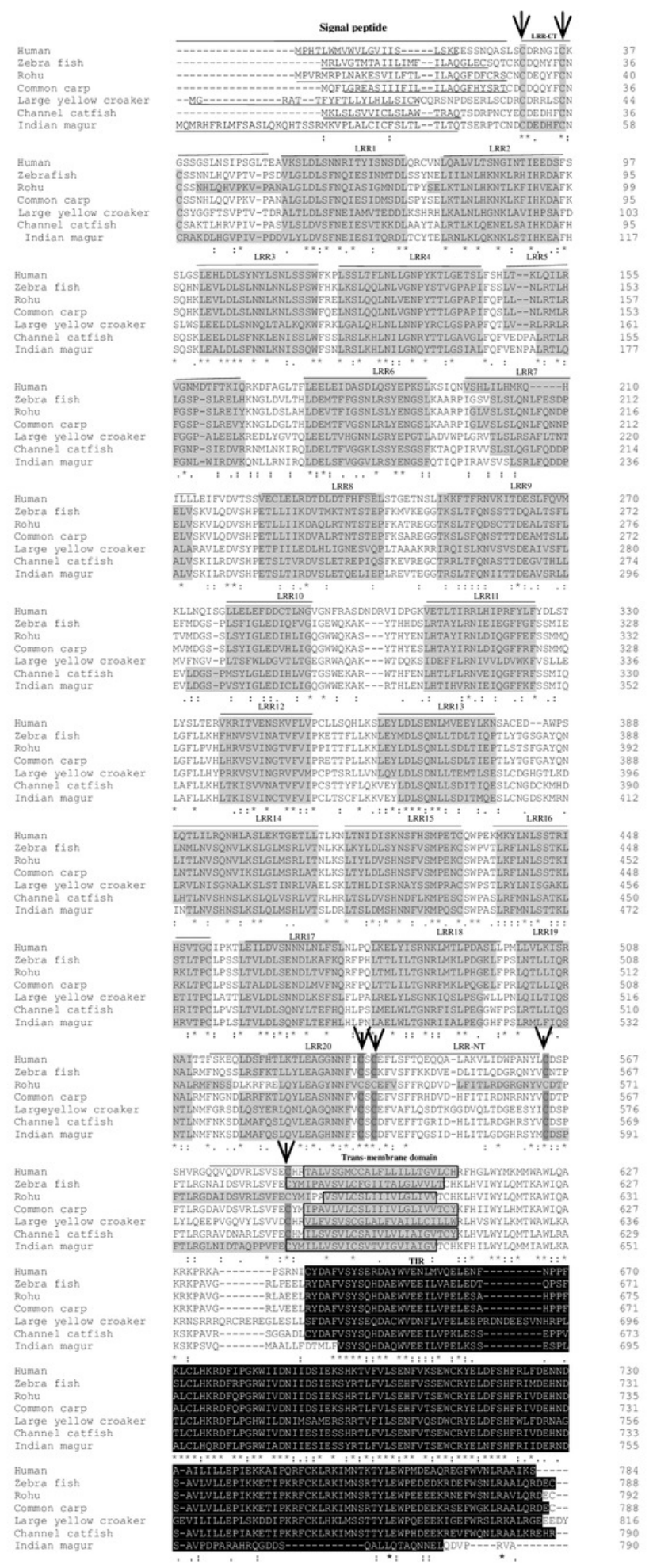




\section{Figure 4}

Phylogenetic relationship of magur $t / r 2$ with other species.

tIr2 amino acid sequences of fishes, human, animals were aligned using CLUSTALW program and the phylogenetic tree was constructed using the neighbour-joining method with the help of MEGA7 software. The branches were validated by bootstrap analysis from 1000 replications, which were represented as bootstrap value in branch nodes. XP_026784977.1 Pangasius Catfish, AlT83001.1 gilt-head bream, ACT64128.1 Emerald rockcod, AFP44842.1 Nile tilapia, AFZ81806.1 striped beakfish, BAD01044.1 Olive flounder, AFG21856.1 miiuycroaker, CCK73195.1 rainbow trout, ADF81060.1 catla, XP_026114714.1 gold fish, AHI59129.1 Mrigal, AG057934.1 crucian carp, AGR53440.1 prussian carp, ACT68333.1 Grass carp, ANI19836.1 Wuchang bream, XP_017572771.1 red bellied piranha, ANA09009.1 yellow catfish, ACT64128.1 Emerald rock cod, ACT64127.1 Antarctic teleost icefish, AEB32453.1 orange-spotted grouper, BAD01046.1 Japanese flounder, AAW69370.1 fugu, ABD17347.1 channel catfish, NP_997977.1 zebrafish, ACP20793.2 common carp, ADQ74644.1 rohu, BAB16113.2 Chicken, AAH14693.1 mouse, NP_942064.1 Rat, NP_001076250.1 Rabbit, AAH33756.1 Human, ADH84419.1 Chimpanzee, NP_001005264.2 Dog, ACH92789.1 Cattle, BAD90590.1 Pig. 


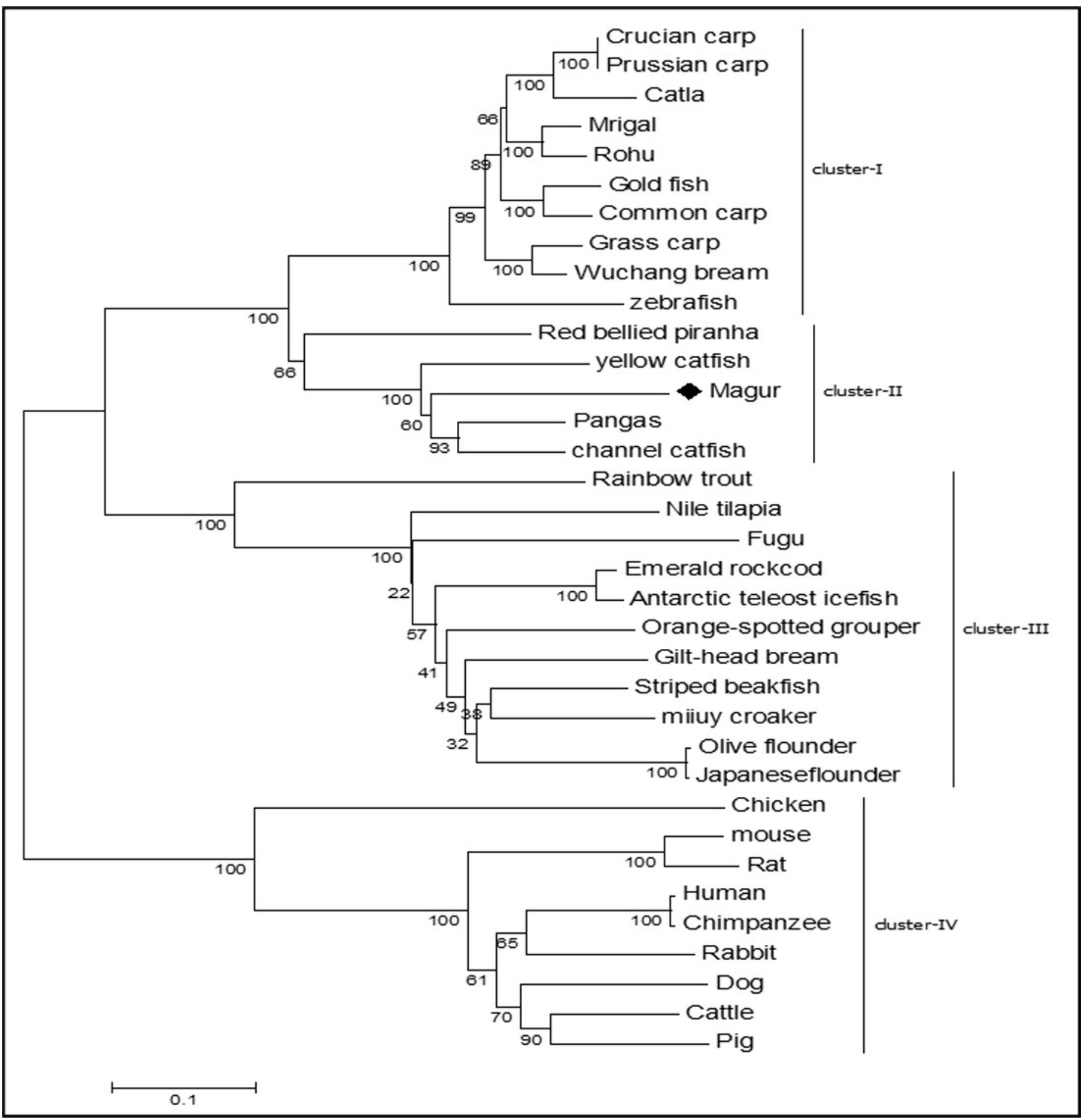




\section{Figure 5}

Basal expression of $m t / r 2(\mathrm{~A}), m y d 88(\mathrm{~B}), n f-k b(\mathrm{C})$ and $i l-1 \beta(\mathrm{D})$ genes in various tissues.

The qRT-PCR was carried out to analyse basal expression of these genes in gill, liver, spleen, intestine, anterior kidney, posterior kidney, stomach, skin, muscle, heart, brain and accessory respiratory organ (ARO). The mRNA transcript levels in various tissues were represented as ratio relative to ef- $1 \alpha$ (internal control). Basal expression in other tissue represented as fold change from calibrator. Skin expressed lowest $m t / r 2$, was used as calibrator and muscle used as calibrator for myd88, nf-kb and il-1 $\beta$. The results were expressed as mean \pm standard error of mean (bars) from three fish $(n=3)$. 

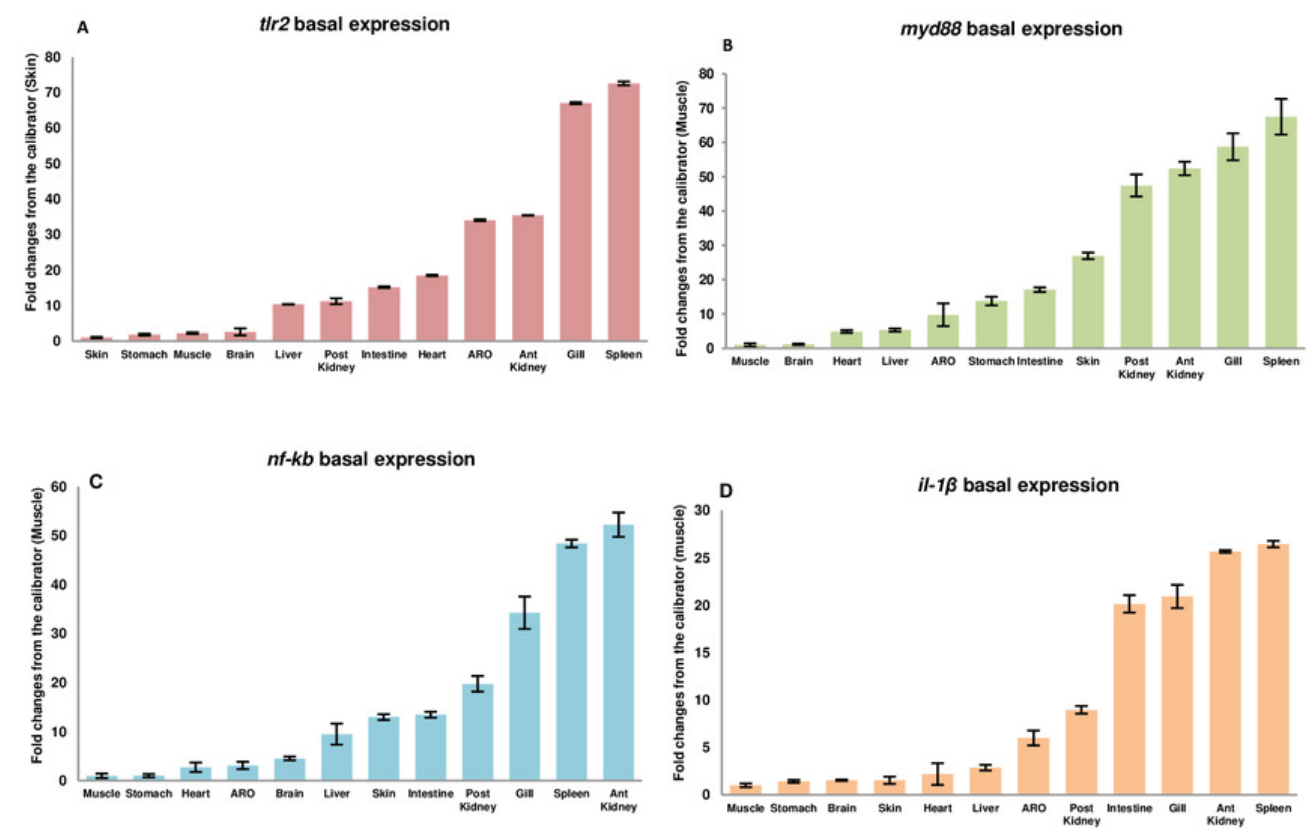


\section{Figure 6}

Modulation of $m t / r 2$ expression following S. aureus infection.

S. aureus ( $1 \times 10^{5} \mathrm{cfu} /$ fish) was injected into magur juveniles by intra peritoneal route. At each experimental time point, total RNA was extracted from gill, liver, spleen, intestine, kidney; qRT-PCR was conducted to analyse the expression of $m t / r 2$. ef-1 $\alpha$ was used as an internal control. The results were expressed as mean \pm standard error (bars) from three fish $(n=3)$. a,b,c: $p$ values significant at $<0.05,<0.01,<0.001$ levels, respectively, compared to controls (one-way ANOVA). 


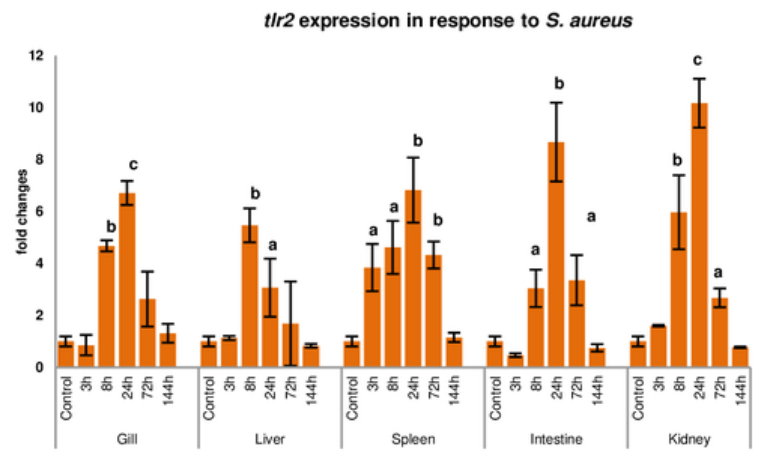




\section{Figure 7}

Modulation of $m t / r 2(\mathrm{~A})$, myd88 (B), $n f-k b(\mathrm{C}), i-1 \beta$ (D) expression following $A$. hydrophila infection.

A. hydrophila( $1 \times 10^{5} \mathrm{cfu} /$ fish $)$ was injected into magur juveniles by intra peritoneal route. At each experimental time point, total RNA was extracted from gill, liver, spleen, intestine, kidney; qRT-PCR was conducted to analyse the expression of $m t / r 2$ and its down-stream signaling molecules. ef- $1 \alpha$ was used as an internal control. The results were expressed as

mean \pm standard error (bars) from three fish $(n=3){ }^{a, b, c}: p$ values significant at $<0.05,<0.01$, $<0.001$ levels, respectively, compared to the controls (one-way ANOVA). 

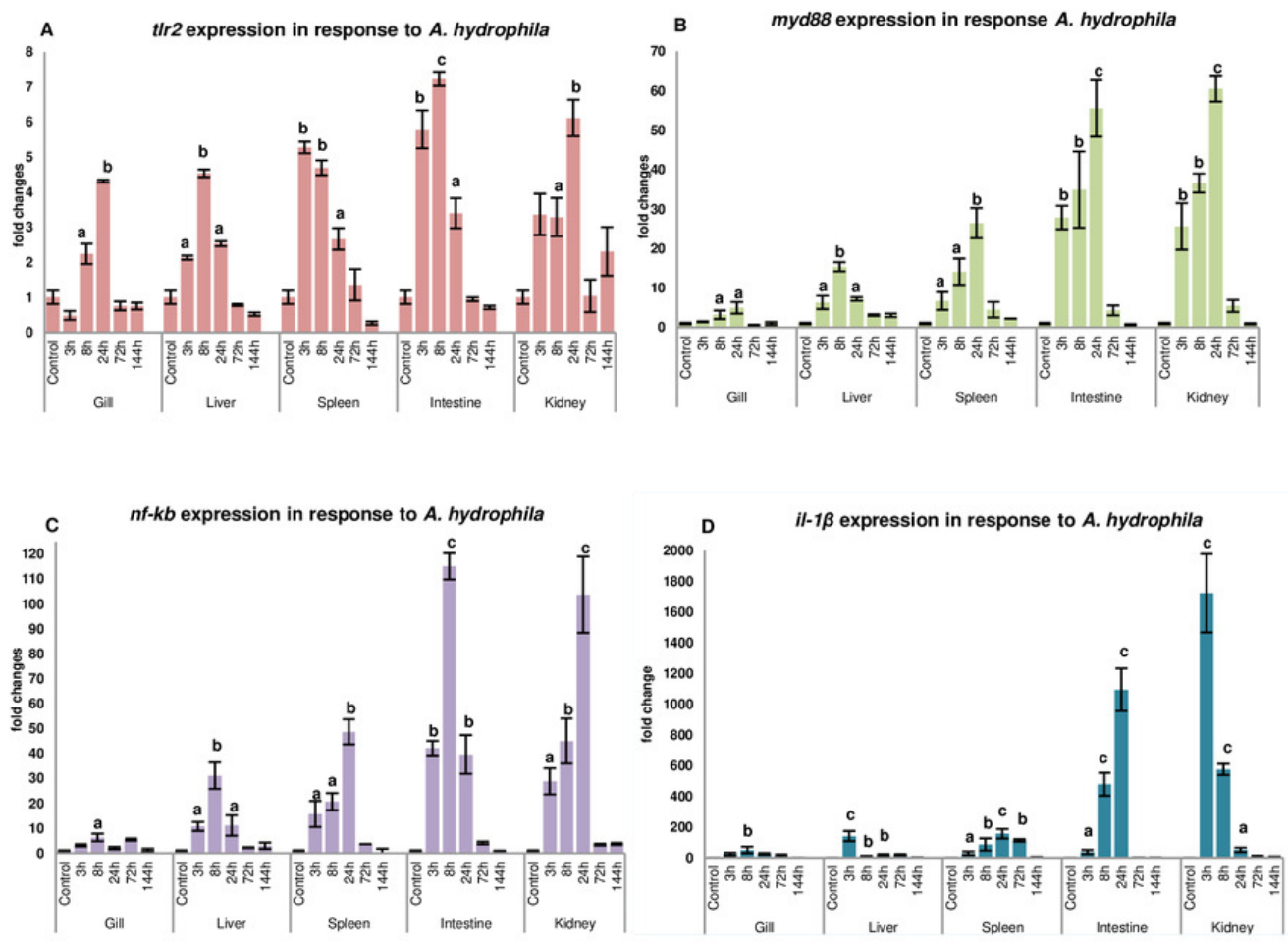


\section{Table $\mathbf{1}$ (on next page)}

Primers, their sequences and their application in this study 
1

\begin{tabular}{|c|c|c|c|}
\hline Primers & Sequence (5'-3') & $\begin{array}{l}\text { GenBank } \\
\text { Accession no }\end{array}$ & Usages \\
\hline \multicolumn{4}{|c|}{ (A)Primers used for PCR amplification and cloning of magur TLR2 } \\
\hline $\operatorname{tlr} 2-\mathrm{F} 1(2 \mathrm{~F})$ & CTGAAACATCTGAACATCTTGG & & $\begin{array}{l}\text { First round homology } \\
\text { PCR }\end{array}$ \\
\hline $\operatorname{tlr} 2-\mathrm{R} 1(3 \mathrm{R})$ & ACGCTAACTGTATCATGCTGC & & \\
\hline $\operatorname{tlr} 2-\mathrm{F} 2(3 \mathrm{~F})$ & CCAGGGCTTCTTCAAATTTAGC & & $\begin{array}{l}\text { First round homology } \\
\text { PCR }\end{array}$ \\
\hline $\operatorname{tlr} 2-\mathrm{R} 2(5 \mathrm{R})$ & TGGACTCGATGATGTTGTCG & & \\
\hline $\operatorname{tlr} 2-\mathrm{F} 3$ & CTTCCAGACGATTCAACCCAT & & Nested PCR \\
\hline $\operatorname{tlr} 2-\mathrm{R} 3$ & GCTCATGTCTAGCGATGTCAG & & \\
\hline T2-5'MR & ACGGCTCTGATGGGTTGAATCGTCTGG & & 5' RACE \\
\hline $\mathrm{T} 2-5^{\prime} \mathrm{MNR}$ & AACGTTCTCAGCGCGGGGTTTTCGAC & & 5' Nested RACE \\
\hline T2-3'MR & GGGTCACCTGCCACAAATTCCACATC & & 3' RACE \\
\hline T2-3'MNR & GCTTTTCGTGTCGTACAGCCAGCAC & & 3' Nested RACE \\
\hline UPM & $\begin{array}{l}\text { Long/CTAATACGACTCACTATAGGGCAAGCAGTGGT } \\
\text { ATCAACGCAGAGT } \\
\text { Short/CTAATACGACTCACTATAGGGC }\end{array}$ & & \\
\hline NUP & AAGCAGTGGTATCAACGCAGAGT & & \\
\hline \multicolumn{4}{|c|}{ (B)Primers used for real-time gene expression analysis } \\
\hline tlr2rt-F & CCAGGGCTTCTTAAATTTAGC & MT625141 & RealtimePCR \\
\hline tlr2rt-R & AGGAGGTTTTGGCTAAGTCC & & \\
\hline$e f-1 \alpha \mathrm{F}$ & GCAGCTTATCGTTGGAGTCA & AB916539.1 & \\
\hline ef-1 $1 \alpha \mathrm{R}$ & GAAATTGGGACGAAAGCAACG & & \\
\hline myd88-F & CTGAAGCTGTGCGTGTTTGA & JQ990986.1 & \\
\hline myd88-R & CTGGAAGTCACAAGCATCGC & & \\
\hline$n f-k b-\mathrm{F}$ & AGAAGAAGGATGTGGCCGAA & MG571500.1 & \\
\hline$n f-k b-\mathrm{R}$ & CATGGTGATGGTTGCTTGGA & & \\
\hline$i l-1 \beta-\mathrm{F}$ & GACCAGGAGCTCTTCAATATCT & JQ309137.1 & \\
\hline$i l-1 \beta-\mathrm{R}$ & AGCCCTTTTAGACTTGTCACA & & \\
\hline
\end{tabular}


Table 2 (on next page)

Identity of magur $t / r 2$ at nucleotide and amino acid level with other fish and animal species 
1

\begin{tabular}{ccccc}
\hline Species & Common name & $\begin{array}{c}\text { mtlr2 } \\
\text { nucleotide } \\
\text { identity (\%) }\end{array}$ & $\begin{array}{c}\text { mtlr2 deduced } \\
\text { aminoacid } \\
\text { Identity (\%) }\end{array}$ & $\begin{array}{c}\text { Tir domain } \\
\text { aminoacid } \\
\text { Identity (\%) }\end{array}$ \\
\hline Pangasionodon hypophthalmus & Pangasius & 83.19 & 77.63 & 89.9 \\
Ictalurus punctatus & Channel catfish & 79.20 & 76.4 & 86.52 \\
Tachysurusfulvidraco & yellow catfish & 80.69 & 72.83 & 87.64 \\
Pygocentrus nattereri & red bellied & 71.38 & 63.98 & 78.65 \\
piranha & & & \\
Labeo rohita & rohu & 66.46 & 59.9 & 82.02 \\
Cirrhinus mrigala & mrigal & 65.76 & 60.2 & 83.15 \\
Megalobrama amblycephala & Wuchang bream & 66.52 & & 80.9 \\
Ctenopharyngodon idella & Grass carp & 66.56 & 60.4 & 82.02 \\
Danio rerio & zebrafish & 58.8 & 58.2 & 77.5 \\
Takifugu rubripes & fugu & 45.6 & 44.1 & 59.3 \\
Oncorhynchus mykiss & rainbow trout & 55.0 & 48.4 & 69.7 \\
Oreochromis niloticus & Nile tilapia & 53.7 & 43.9 & 57.9 \\
Paralichthys olivaceus & Japanese & 46.6 & 42.9 & 57.4 \\
& flounder & & & \\
Chionodraco hamatus & Antarctic teleost & 53.7 & 46.2 & 59.4 \\
Homo sapiens & icefish & & & 65.2 \\
Gallus gallus & Human & 51.3 & 38.7 & 64 \\
Bos taurus & Chicken & 50.1 & 37.3 & 64 \\
Canis lupus familiaris & Cattle & 51.4 & 38.4 & 65.2 \\
Oryctolagus cuniculus & Dog & 52.3 & 39 & 65.2 \\
Mus musculus & Rabbit & 51.0 & 38.4 & 64 \\
\hline
\end{tabular}

2 


\section{Table 3 (on next page)}

The amplification efficiencies $(E)$ and correlation coefficient $\left(R^{2}\right)$ of qRT-PCR primers used in this study 
1

\begin{tabular}{ccc}
\hline Symbol & Amplification Efficiencies (\%) & Correlation coefficient $\left(\mathrm{R}^{2}\right)$ \\
\hline$t l r 2$ & 104.66 & 0.998 \\
$m y d 88$ & 102.45 & 0.989 \\
$n f-k b$ & 97.94 & 0.989 \\
$i l-1 \beta$ & 95.81 & 0.996 \\
$e f-1 \alpha$ & 99.25 & 0.998 \\
\hline
\end{tabular}

2

3

4 